\title{
Monitoring atmospheric phenomena on Titan
}

\author{
M. Hirtzig ${ }^{1}$, A. Coustenis ${ }^{1}$, E. Gendron ${ }^{1}$, P. Drossart ${ }^{1}$, A. Negrão ${ }^{1,3}$, M. Combes $^{1}$, O. Lai ${ }^{1,2}$, P. Rannou $^{3}$, \\ S. Lebonnois ${ }^{4}$, and D. Luz ${ }^{1}$ \\ 1 LESIA, Observatoire de Paris-Meudon, 92195 Meudon Cedex, France \\ e-mail: mathieu.hirtzig@obspm.fr \\ 2 CFHT, Hawaii \\ 3 Service d'Aéronomie/IPSL, Univ. Versailles St-Quentin, France \\ ${ }^{4}$ LMD Jussieu, 75252 Paris Cedex 05, France
}

Received 6 May 2005 / Accepted 10 February 2006

\begin{abstract}
For the past 8 years (1998-2005), we have used adaptive optics imaging (with VLT/NACO and CFHT/PUEO) to explore Titan's atmosphere, which is currently scrutinized in situ by the Cassini-Huygens mission. In the course of our work, we have found variations, such as as seasonal and diurnal effects, as well as temporary features in the southern polar region. The north-south asymmetry is shown to have changed since 2000 in the near-IR and to be currently organized in a brighter northern than southern pole. We study this evolution here. With our data, we also have new significant statistical evidence of diurnal effects in Titan's stratosphere, with a brighter (as much as 19\%) morning limb appearing in our images in many cases, when the phase effect is expected on the evening side. The southern bright feature is probably a time-limited seasonal and/or meteorological phenomenon, revolving around the south pole (confined in its motion within the $80^{\circ} \mathrm{S}$ parallel) and located somewhere in the upper troposphere (18-40 km of altitude). Its behavior and possible nature are discussed here.
\end{abstract}

Key words. planets and satellites: individual: Titan - instrumentation: adaptive optics

\section{Introduction}

We observed Titan with five different adaptive optics systems from 1994 to 2005, monitoring surface and atmospheric features (Combes et al. 1997; Coustenis et al. 2001; Gendron et al. 2004; Hirtzig et al. 2005; Coustenis et al. 2005). In the past 8 years we have performed imaging with NAOS/CONICA (NACO) at the Very Large Telescope (Yepun, 8-m, Chili) and with PUEO at the Canada France Hawaii Telescope (3.6-m, Hawaii) in order to characterise the surface and the atmosphere of the satellite. The use of adaptive optics is compulsory for the study of this 0.8 -arcsec wide target, if we are to resolve the features on Titan's disk. Our findings in terms of surface properties are described in Coustenis et al. (2005). In this paper we focus on the Titan atmospheric phenomena by investigating the chronological variations in the features found in our images.

Titan's near-infrared spectrum contains a number of strong methane bands probing the atmosphere, and in between them, specific regions are located where the methane absorption is weak enough to allow the retrieval of information on the surface: these can be found at 1.08, 1.09, 1.28, 1.6, 2.0, 2.75, and $4.9 \mu \mathrm{m}$. The filters we used to probe Titan's atmosphere and surface at different altitudes are described in detail in the CFHT and VLT web sites and are also listed in Table 3 of Coustenis et al. (2005). These authors have studied Titan with surface filters (the ones including contribution from the surface and centered in the methane windows at $1.08,1.09,1.28,1.6$, and $2.0 \mu \mathrm{m}$ ) showing bright and dark features on both hemispheres.

\subsection{Observations}

Table 1 lists our observations of the Titan atmosphere. We added up our images taken on the same day in a given filter to recover the best signal-to-noise ratio (SNR) possible. The NACO dates correspond to Titan angular radii of 0.44 for 2002 and 2005, and 0.37 for 2004 . The PUEO data correspond to apparent radii of $0.37,0.43$, and 0.44 for 2001,2002 , and 2004, respectively. This explains the variations in Titan's apparent size shown in the figures herein, as for instance in Fig. 1, even though Titan is observed with the same instrument.

Described in the logbook (Table 1) are some of the observational restrictions we had to face during our campaigns. For instance, NACO images occasionally suffered from poor seeing conditions. Indeed the seeing was around 2 arcsec in 2002, with optimal conditions (around 1 arcsec) on the last night of observing. In 2004, the seeing was improved, but the wind was too strong to allow us to acquire more than a few images, albeit with a good seeing of about 0.6 arcsec. Finally, the 2005 NACO images benefited from good $(0.4-0.6$ arcsec) seeing. In comparison, all PUEO images prior to 2004 were taken with good and stable seeing conditions, on the order of 0.6 arcsec during all of our observational runs, sometimes even reaching 0.4 (14 November 2002).

An example of poor observing conditions is our January 2004 data, when we faced a serious problem with PUEO/KIR, as the telescope seemed to drift under its own weight. This drift caused our images to be somehow blurred, and it was a challenge to compensate for this effect with the right PSF in view of an efficient processing of our data. 
Table 1. Titan adaptive optics observations from 1998 to 2005 with CFHT/PUEO and VLT/NACO.

\begin{tabular}{|c|c|c|c|c|c|}
\hline Date (UT) & Inst. & Filters & Solar phase angle & $\overline{\text { SEP LCM }}$ & Seeing \\
\hline 26 Oct. 1998 & PUEO & $\mathrm{J} 1, \mathrm{~J} 2, \mathrm{H} 1, \mathrm{H} 2$ & $+0.51^{\circ}$ & $93-97^{\circ}$ & $0.3-0.5$ \\
\hline 07 Mar. 2001 & PUEO & J2, J1, Jcont, H1, Hcont, Kcont & $+5.83^{\circ}$ & $85-87^{\circ}$ & $0.8-1.0$ \\
\hline 08 Mar. 2001 & PUEO & I, PaGam, HeI, H1, Hcont, FeII & $+5.80^{\circ}$ & $108-110^{\circ}$ & $1.0-1.2$ \\
\hline 04 Dec. 2001 & PUEO & H1, FeII, Jcont, J1, J2, HeI, PaGam, K, K', H2(1-0) & $+0.29^{\circ}$ & $112-119^{\circ}$ & $0.5-0.8$ \\
\hline 13 Nov. 2002 & PUEO & BrGam, H2(1-0), FeII, H2, J2, J1, H1 & $-3.78^{\circ}$ & $283-287^{\circ}$ & $0.6-0.8$ \\
\hline 14 Nov. 2002 & PUEO & J1, J2, H1, H2, FeII, BrGam, H2(1-0) & $-3.68^{\circ}$ & $305-309^{\circ}$ & $0.3-0.8$ \\
\hline 20 Nov. 2002 & NACO & $\mathrm{NB} \_1.08, \mathrm{NB} \_1.24, \mathrm{NB} \_1.28, \mathrm{NB} \_1.64, \mathrm{NB} \_1.75, \mathrm{NB} \_2.12, \mathrm{NB} 2.17$ & $-3.13^{\circ}$ & $81-82^{\circ}$ & $1.7-2.3$ \\
\hline 20 Nov. 2002 & PUEO & J1, J2, H1, H2, FeII, BrGam, H2(1-0) & $-3.11^{\circ}$ & $83-85^{\circ}$ & $0.7-1.0$ \\
\hline 21 Nov. 2002 & PUEO & $\mathrm{J} 1, \mathrm{~J} 2, \mathrm{H} 1, \mathrm{H} 2$, FeII, BrGam, H2(1-0) & $-3.00^{\circ}$ & $103-107^{\circ}$ & $0.5-0.8$ \\
\hline 25 Nov. 2002 & $\mathrm{NACO}$ & NB_1.08, NB_1.09, NB_1.24, NB_1.28, NB_2.12, NB_2.17 & $-2.60^{\circ}$ & $189-191^{\circ}$ & $1.3-1.6$ \\
\hline 26 Nov. 2002 & NACO & $\mathrm{NB} \_1.08, \mathrm{NB} \_1.24, \mathrm{NB} \_1.28, \mathrm{NB} \_1.64, \mathrm{NB} \_1.75, \mathrm{NB} \_2.12, \mathrm{NB} 2.17$ & $-2.49^{\circ}$ & $211-214^{\circ}$ & $0.9-1.0$ \\
\hline 07 Jan. 2004 & PUEO & FeII, H2(1-0), H2, H1, $\overline{\mathrm{Pa}} \beta, \mathrm{J} 2$ & $+0.80^{\circ}$ & $11-13^{\circ}$ & $0.7-1.0$ \\
\hline 08 Jan. 2004 & PUEO & $\mathrm{H} 2(1-0), \mathrm{BrGam}, \mathrm{K}, \mathrm{K}^{\prime}, \mathrm{J} 1, \mathrm{~J} 2, \mathrm{~J}, \mathrm{~Pa} \beta$, Jcont & $+0.91^{\circ}$ & $31-34^{\circ}$ & $0.4-0.6$ \\
\hline 25 Apr. 2004 & $\mathrm{NACO}$ & IB_2.00, NB_2.12, IB_2.15, NB_2.17 & $+5.65^{\circ}$ & $344-346^{\circ}$ & $0.5-0.6$ \\
\hline 16 Jan. 2005 & NACO & NB_1.28, IB_2.00, NB_2.12, IB_2.15, NB_2.17 & $+0.24^{\circ}$ & $192-194^{\circ}$ & $0.4-0.7$ \\
\hline
\end{tabular}

Therefore, our 2004 PUEO images are unreliable unless we can test them independently.

The atmospheric filters probe different altitude levels and are centered in the methane bands (at 1.04, 1.24, 1.64, 1.75, 2.12, and $2.17 \mu \mathrm{m}$ - see Table 2 for altitude range and description of filter widths). Some filters contain both surface and atmosphere contributions (NB_1.04, NB_1.08, NB_1.28, H1, Pa $\beta, \mathrm{J} 1$, Hcont, IB_2.00), but note that in this work we mainly consider the ones probing the atmosphere.

\subsection{Data processing}

The processing applied to the data here is similar to the one described in Combes et al. (1997), Coustenis et al. (2001), and Coustenis et al. (2005), namely flatfielding and bad pixel correction, followed by deconvolution using standard photometric stars as PSF references. This treatment returned diffraction-limited images in most cases when we used the Magain deconvolution. However, sometimes we had to choose lower-resolution deconvolution methods (as for instance the Bratsolis \& Sigelle (2001) version of the Richardson-Lucy (RL) method) for cases of extremely poor seeing, as was the case for our March 2001 and January 2004 PUEO images, in particular. We took great care to use only one method for a given series of observations, so as to ensure that comparisons within one night are still valid and suffer mainly from systematic errors (which are not a problem when we discuss relative measurements). Exceptions are the 1998 and January 04 PUEO series, which we deconvolved here ${ }^{1}$ with the Bratsolis and Sigelle RL method (see Sect. 3.1 of Coustenis et al. (2005) for more details on the deconvolution processes and the justification for choosing only one of them).

Note that for this study we chose not to correct for centerto-limb effects, in contrast to Coustenis et al. (2005). Since we focus here only on the study of Titan's limb (or a one-resolutionelement-thick ring on Titan's limb), the limb-to-limb effects are indeed more significant than in the study of the limb of Titan's disk. The correction is far more sensitive to the application of a cylindrical filter (definition of the disk's radius and limb) near the limb than in the limb. If a cylindrical mask is shifted by only one pixel from the real position of Titan's disk limb, then the flux variation on the limb can easily reach $40 \%$. The same occurs if the cylindrical mask radius is one pixel larger

${ }^{1}$ In Coustenis et al. (2001), we used the MISTRAL deconvolution, which we did not use here to keep only a small number of deconvolution methods, so as to be able to make valid comparisons. or smaller than Titan's real radius. All this would lead to additional errors, and since we will only consider relative comparisons hereafter, we did not correct for limb-to-limb effects, studying only a one-resolution-element-thick group of pixels on the disk limb (from about 70 to $90^{\circ}$ from the nadir).

A similar problem arises regarding the "ringing effect" due to deconvolution. This phenomenon (also known as "Gibbs' ring") artificially enhances the brightness of the limbs after deconvolution because of the proximity of a high-luminosity gradient between Titan's edge and the dark sky in the background. This might induce additional errors in our computations. In fact, we can assume that the ringing effect is equal for all the pixels at a given distance from Titan's center. As a matter of fact, the Gibbs' ring cannot be omitted when comparing pixels in the center of the disk with pixels on the limbs, for instance. But here, we only build relative comparisons between pixels on the limbs exclusively, and the ringing will thus be cancelled in our ratio calculations.

All the intensity values presented here correspond to an averaged value of the $2 \times 2$ (near $1 \mu \mathrm{m}$ ) or $3 \times 3$ (near $2 \mu \mathrm{m})$ CCD pixels that represent the actual size of one resolution element as recorded on the detector. The position of the limbs is defined by the location of the center of Titan's disk and the extent of its radius. The latter is computed from the Institut de Mécanique Céleste et de Calcul des Ephémérides (www.imcce.fr/imcce.php) tables which return the angular size of the satellite on any given date, divided by the angular size of the detector $(0.0348$ and $0.01325 \mathrm{arcsec} / \mathrm{pix}$ for PUEO and NACO respectively). The center of Titan's disk is found with a 0.4 CCD-pixel precision as follows:

First we assume that Titan's bulk is similar to a 3D Gaussian, and for this we suppress all the variable features to recover a smoothed uniform Titan's disk; we consider that all these features (whether they are linked to surface structures or atmospheric phenomena, like the limb-brightening, for instance) are found in the $20 \%$ upper luminosity range, and we then consider this threshold as an upper limit for the definition of Titan's bulk. In this process, we thus transform Titan's disk into a 3D bellcurve with a "plateau" on the top (the late erased brightest features on the disk). Once Titan's features are erased, we fit the remnant of the shape with a 3D Gaussian, given three adaptable parameters: height, FWHM, and center of the Gaussian shape. The FWHM returns the diameter of the object, and the center coordinates correspond to Titan's central ones. The error bar on this 


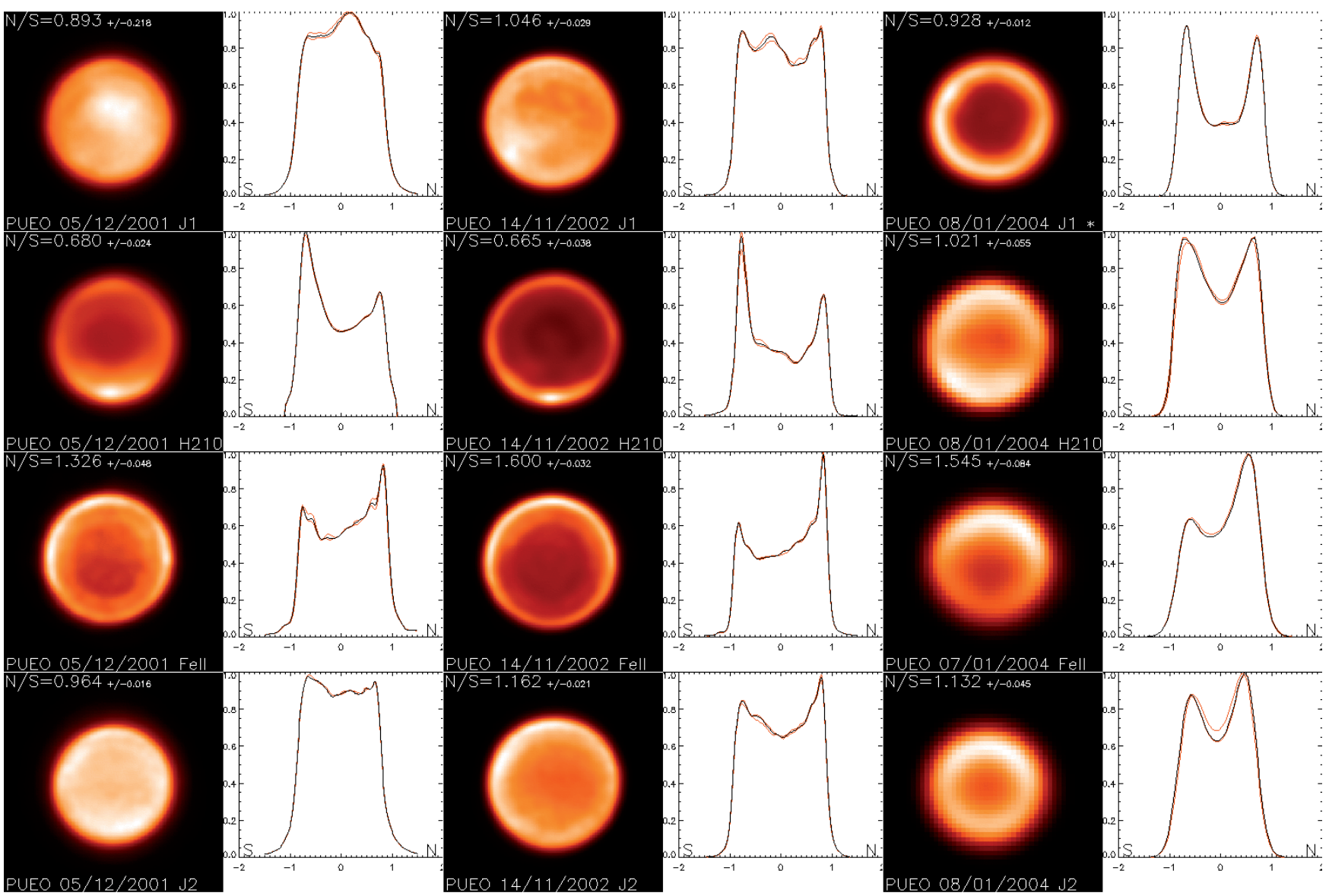

Fig. 1. Typical PUEO images showing the evolution of the north-south asymmetry (NSA) on Titan as a function of altitude (different levels are probed in the different atmospheric filters) and time. Altitude decreases vertically (the filters are the same across each horizontal set), with the surface probed at the top and the highest layers at the bottom; time increases from December 2001 (left panel) to January 2004 (right panel). On each image, north is up, and west-map is to the left. The profiles drawn correspond to normalized intensity ( 1 being the brightest resolution element of the whole image) from one limb to another. Error bars, shown in orange lines, correspond to variations from one resolution element to another. These profiles provide the north-south contrast with good precision, as indicated in the upper left corner of each sub-image. Many other features are visible in these images. First, the ringing effect in all the 2004 images (right column) is due to a poor deconvolution correction caused by poor observing conditions. All the other data return better corrected images, on which we can notice bright northern or southern limbs (involved in what we called north-south asymmetry, see Sect. 2), different behaviors of the eastern and western limbs (the east-west asymmetry, interpreted here as a diurnal phenomenon, are described in Sect. 3), and some bright features near Titan's south pole (particularly at $2.12 \mu \mathrm{m}$, on the second horizontal panel, as we develop in Sect. 4). The surface of Titan is also visible on the upper panel images, with the bright continent "Xanadu" protruding on the leading hemisphere of Titan (observed in 2001), or the bright and dark patches dispatched across the trailing hemisphere (observed in 2002).

fit is taken into account for positioning issues, but it is too high to ensure a safe limb-to-limb correction, as explained earlier.

\subsection{Radiative transfer model}

In order to be efficient, any study of atmospheric images requires a complete radiative transfer model to compute altitude information for each filter used. We used the microphysical and radiative transfer code of Rannou et al. (2003), which is an updated version of the one described in McKay et al. (1989). This model assumes that the aerosols in Titan's atmosphere are fractal-shaped (Rannou et al. 1995). The haze profile and all the parameters for this model are fully described in Rannou et al. (2003), where the sensitivity of the model to the different parameters can also be found. However, in Coustenis et al. (2005) and the work herein, we used the updated parameter values proposed by Negrão et al. (2006) and Coustenis et al. (2006). These updated parameters were derived from a work based on ISO (for Infrared Space Observatory) and ground-based nearinfrared measurements (from 1 to $2.5 \mu \mathrm{m}$ ). The alterations, compared with Rannou et al. (2003), consist in an overall increase of $40 \%$ in the haze density vertical profile, as calculated by the microphysical part of the model. Furthermore, although the altitude of the haze cut-off applied in this model remains the same as the one used in Rannou et al. (2003), the shape of the haze vertical profile below the cut-off is slightly different. A new methane abundance near the surface of $5 \%$ - instead of the $8 \%$ that was assumed prior to the recent Cassini measurements - is consistent with the recent disc-averaged DISR observations (Tomasko et al. 2005), was used in these calculations. The $\mathrm{CH}_{4}$ mixing ratio remains constant from the surface up to the saturation level, then follows the saturation value until the tropopause, above which it stays constant. The methane absorption coefficients used in this model are taken from a re-analysis of K. Strong (Strong et al. 1993) and K. Sihra (Sihra 1998) data by P. Irwin (Irwin et al. 2006, Sromovsky et al. 2006) for the 1.08 and $1.28 \mu \mathrm{m}$ windows, and from the Hilico et al. (1994) updated database at and beyond $1.6 \mu \mathrm{m}$ (Boudon et al. 2006). For an update on this model and recent results, see Coustenis et al. (2006) and Negrão et al. (2006). 
Table 2. N/S ratio characterizing the north-south asymmetry from 1998 to 2005 . We also show the altitudes probed at the limb by the different filters used.

\begin{tabular}{|c|c|c|c|c|c|c|c|c|}
\hline \multirow[t]{2}{*}{$\overline{\overline{\text { Filter }}}$} & \multirow{2}{*}{$\begin{array}{c}\text { Wavelength } \\
(\mu \mathrm{m})\end{array}$} & \multicolumn{2}{|c|}{ Alt limb } & \multicolumn{5}{|c|}{ N/S ratio } \\
\hline & & $\operatorname{mini}(\mathrm{km})$ & $\operatorname{maxi}(\mathrm{km})$ & 1998 & 2001 & 2002 & 2004 & 2005 \\
\hline NB_1.04 & $1.040 \pm 0,007$ & 36 & 43 & - & - & $1.019 \pm 0.005$ & - & - \\
\hline NB_1.09 & $1,094 \pm 0,0075$ & 6 & 18 & - & - & $0.987 \pm 0.004$ & - & - \\
\hline$\overline{\mathrm{Pa} \gamma}$ & $1,094 \pm 0,005$ & 5 & 21 & - & $0.872 \pm 0.015$ & - & - & - \\
\hline $\mathrm{J} 2$ & $1,181 \pm 0,064$ & 58 & 155 & $0.883 \pm 0.089$ & $0.964 \pm 0.016$ & $1.162 \pm 0.021$ & $1.132 \pm 0.045$ & - \\
\hline Jcont & $1,207 \pm 0,007$ & 43 & 65 & - & $0.877 \pm 0.018$ & - & $1.043 \pm 0.048$ & - \\
\hline NB_1.24 & $1,237 \pm 0,0075$ & 23 & 45 & - & - & $0.974 \pm 0.011$ & - & - \\
\hline NB_1.28 & $1,282 \pm 0,007$ & 1 & 3 & - & - & - & - & - \\
\hline $\mathrm{Pa} \beta$ & $1,282 \pm 0,007$ & 1 & 3 & - & - & - & - & - \\
\hline $\mathrm{J} 1$ & $1,293 \pm 0,070$ & 0 & 58 & - & - & - & - & - \\
\hline Hcont & $1,570 \pm 0,010$ & 8 & 9 & - & - & - & - & - \\
\hline $\mathrm{H} 1$ & $1,600 \pm 0,080$ & 9 & 104 & - & - & $1.027 \pm 0.021$ & $0.864 \pm 0.042$ & - \\
\hline NB_1.64 & $1,644 \pm 0,009$ & 45 & 322 & - & - & $1.549 \pm 0.015$ & - & - \\
\hline $\mathrm{H} 2$ & $1,640 \pm 0,050$ & 21 & 322 & $0.812 \pm 0.094$ & - & $1.102 \pm 0.009$ & $0.927 \pm 0.049$ & - \\
\hline FeII & $1,644 \pm 0,007$ & 45 & 280 & - & $1.326 \pm 0.048$ & $1.600 \pm 0.032$ & $1.545 \pm 0.084$ & - \\
\hline NB_1.75 & $1.748 \pm 0,013$ & 47 & 318 & - & - & $1.091 \pm 0.012$ & - & - \\
\hline IB_2.00 & $2,000 \pm 0,030$ & 9 & 158 & - & - & - & $1.111 \pm 0.036$ & $1.328 \pm 0.012$ \\
\hline NB_2.12 & $2,120 \pm 0,010$ & 17 & 20 & - & - & $0.623 \pm 0.008$ & $1.068 \pm 0.034$ & $1.216 \pm 0.023$ \\
\hline $\mathrm{H} 2(1-0)$ & $2,122 \pm 0,010$ & 17 & 24 & - & $0.683 \pm 0.028$ & $0.665 \pm 0.038$ & $1.021 \pm 0.055$ & - \\
\hline IB_2.15 & $2.150 \pm 0,030$ & 19 & 314 & - & - & - & $1.160 \pm 0.013$ & $1.350 \pm 0.012$ \\
\hline $\mathrm{Br} \gamma$ & $2.166 \pm 0,010$ & 40 & 359 & - & - & $1.333 \pm 0.034$ & $1.453 \pm 0.122$ & - \\
\hline NB_2.17 & $2.166 \pm 0,010$ & 40 & 359 & - & - & $1.091 \pm 0.012$ & $1.357 \pm 0.036$ & $1.653 \pm 0.027$ \\
\hline
\end{tabular}

Titan has a dense and extended atmosphere, so the viewing geometry is a parameter to be taken into account in our modeling. Although the model we use is indeed plane parallel, we implemented a geometrical correction that allows us to calculate the emission angle as a function of altitude. This correction is important since the difference between the emission angle at high altitudes and lower altitudes is more noticeable near the limb, and this difference is important in the radiative transfer calculation. The corrected plane parallel model was then compared with a 3D spherical Monte-Carlo model produced by Tran \& Rannou (2004) and shown to provide a good approximation.

This model computes three altitude calculations per filter depending on the phase angle considered (here we study only the limb, $80^{\circ}$ away from the nadir). The projection of a resolution element on the limb is more or less important, depending on the Earth-Titan distance. As an average: PUEO: limb = 55-90 deg; NACO: limb $=70-90$ deg (Titan's disc is smaller when viewed with PUEO and the projection angle diverges rapidly on the limb). The nominal altitude (used only in Sect. 4) corresponds to the average $\tau_{\text {eff }}=1$ level over the bandwidth of the filter $\left(\tau_{\text {eff }}\right.$ being defined as the effective opacity, which takes the forward scattered photons into account and whose mathematical definition is:

$\left.\tau_{\text {eff }}=\tau \times \sqrt{(}(1-w) \times(1-w \times g)\right)$

where $\tau$ is the opacity, $w$ the single scattering albedo, and $g$ the asymmetry factor (Rannou et al. 2003; Pollack \& McKay 1985). This altitude gives the lowest level probed by the penetrating photons, the effective opacity parameter, taking into account the photons scattered forward by the haze. Because of the significant variation in the methane absorption coefficients within a methane window, these altitudes may change depending on the wavelength; thus we need to consider the minimum and maximum $\tau_{\text {eff }}=1$ layers over the FWHM of a filter, as given in Table 2, which give a good thickness estimate of the layer probed. Note that a narrow-band filter will not necessarily probe a very specific layer if it covers a region where methane absorption coefficients vary quickly; see Coustenis et al. (2006), where the methane coefficients are shown and one can see how, for instance, the NB_2.17 and NB_1.24 NACO filters - though with similar FWHM - probe the 40-300 and 46-50 km ranges respectively. This is because the former spans a wider altitude range because it overlays large methane coefficient variations.

\section{Evolution of the north-south asymmetry on Titan}

Several features are to be found on the limb of Titan, as illustrated in Fig. 1. Some of the earlier infrared images probing the atmosphere (see Table 2) show the well-known bright south pole of Titan. However, since 2001 we have observed an inversion of the north-south asymmetry in some of these filters (at 1.64, 1.75, $2.17 \mu \mathrm{m})$, with a currently bright north pole. Some evidence of an NSA inversion in the visible has been previously provided, as described in Lorenz et al. (1999). This is an expected return to the Voyager situation, predicted by seasonal models (Sromovsky et al. 1981; Hutzell et al. 1993), where the south limb was bright in the visible, but dark in the infrared, anti-mirroring the northern limb situation. This situation had reversed in the 90's, showing the famous "Titan's smile" in the adaptive optics and HST images (see for instance Saint-Pé et al. 1993 and Caldwell et al. 1992, respectively). In some cases, such as at 1.04, 1.09, and $1.24 \mu \mathrm{m}$, we find currently components of both tendencies to be present, with both the northern and the southern hemispheres bright.

In Figs. 1 and 2, we illustrate the NSA evolution as a function of altitude probed by different filters. We quantify the northsouth contrast by computing the ratio of the $\mathrm{N}$ intensity over the $\mathrm{S}$ one, as reported in Table 2 . We consider a one-resolutionelement-thick line following Titan's rotation axis, and we define the southernmost and northernmost resolution elements as representative of the southern and northern limbs. The average flux for these two resolution elements is then used for the computation of the $\mathrm{N} / \mathrm{S}$ ratio. We take the viewing geometry into account (as detailed in Table 1) to ensure that the profiles drawn in Fig. 2 truly intersect the south Pole and centre of Titan's disk. 

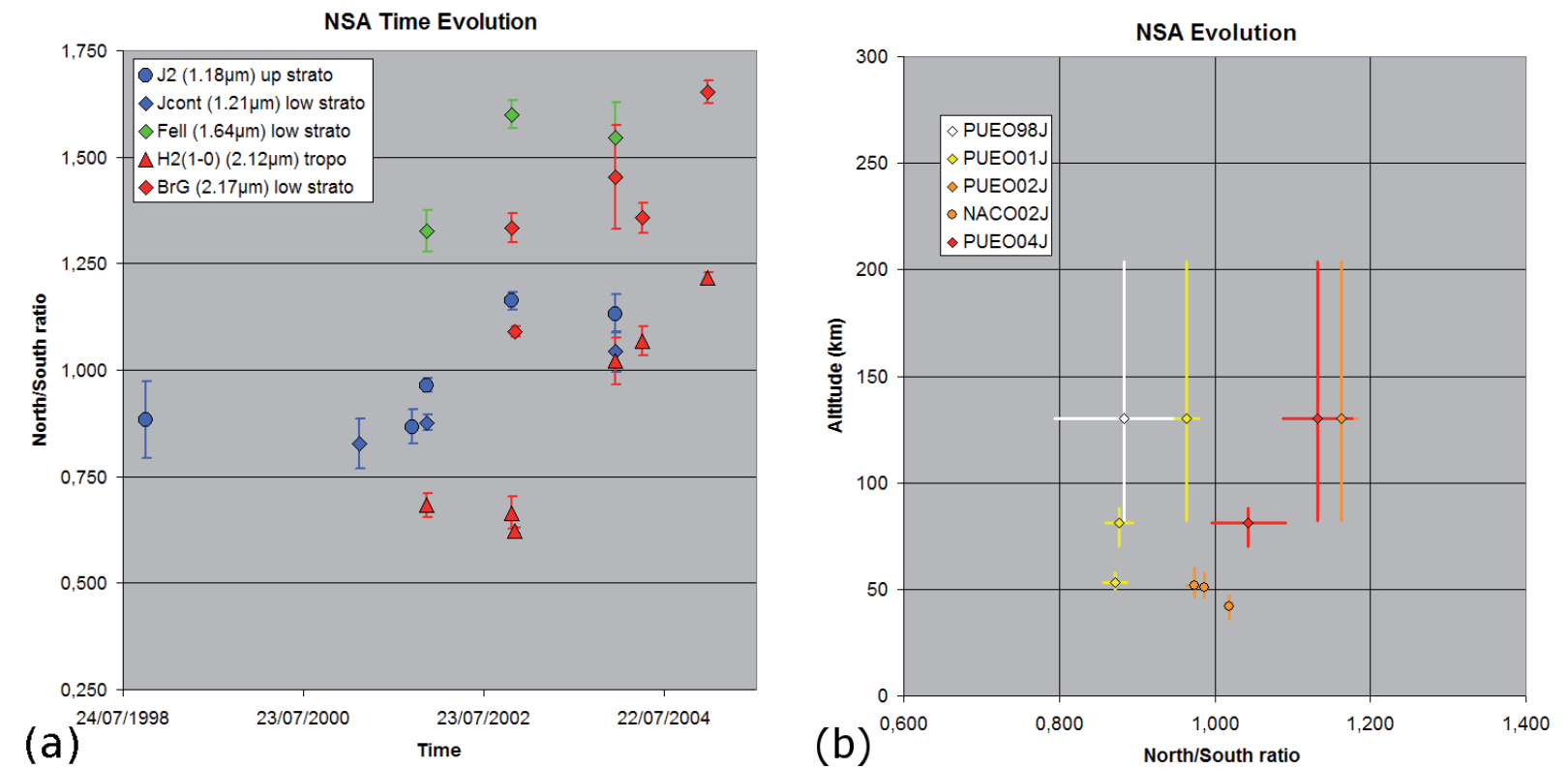

Fig. 2. Figure showing the evolution of the NSA over the past 7 years (1998-2005). Left panel a): time evolution of the NSA with characteristic filters $(1.18,1.21,1.64,2.12$ and $2.17 \mu \mathrm{m}$ ). Colors corresponds to wavelength (blue $=J$ band, green $=H$ band, red $=K$ band), and symbols to altitude (triangles for the troposphere, diamonds for the tropopause, circle for the stratosphere). We combine here both NACO and PUEO data when the corresponding filters are equivalent (e.g. H2(1-0) and NB_2.12) Right panel b): NSA evolution as a function of time and altitude, drawn only for $J$ band filters for legibility; the color code indicates the year of observation ( white $=1998$, orange $=2001$, red $=2002$, black $=2004$ ) while the symbol reflects the instrument used (diamond $=$ PUEO, circle $=$ NACO).

Error bars arise mainly from the variation of the intensity on the limb from one resolution element to another. For this study, we only considered "uniform" and feature-free limbs to avoid deconvolution artifacts. We did not try, for instance, to correct for the remaining ringing effect, because a mere centre-to-limb effect compensation might perhaps introduce dubious results. In particular, the case of the $2.12 \mu \mathrm{m}$ filter is used only as a confirmation and not as a detection of NSA variations; as we will describe in Sect. 4.2, the southern limb in the $2.12 \mu \mathrm{m}$ images is not uniform and continuous, showing many features that cannot be reasonably or efficiently eliminated in order to compute a true average intensity for the southern limb.

In Figs. 1 and 2, we find that there is a definite evolution with Time. The "Titan smile" has faded in most of the 2004-2005 images, and the north limb appears brighter than the southern one in more and more filters as time goes by. Our study indicates that this evolution is a function of the altitude levels probed and that the NSA reverses, while this reversal propagates to lower altitudes with time. In other words, starting out at higher levels in the atmosphere, a bright northern pole replaces the southern one, as with time the reversal affects lower altitudes.

- Variations as a function of time: Figs. 1 and 2a show clearly that a given filter showing the NSA witnesses its reversal with higher and higher north/south ratios as time goes by; for instance, the PUEO J2 filter (blue circles) shows such a trend from 1998 until 2005 (within error bars, the NSA seems not to evolve much between 2002 and $2004^{2}$, but we cannot determine if this is real or artificial). In 2002, the famous "Titan smile" is but a memory; in 2004, all images show a bright northern limb.

${ }^{2}$ In 2004, we suffered from mechanical problems, rendering the analysis PUEO 2004 data difficult; furthermore, all $2.12 \mu \mathrm{m}$ images could not be used in this study (as explained previously).
- Variations as a function of altitude: Figs. 1 and $2 b$ also confirm that the NSA evolves differently depending on the altitude probed. At a given date, the NSA appearance is not the same in all the filters. For instance, in 2001, the Pa $\gamma$ filter (at $1.094 \mu \mathrm{m}$, probing the troposphere) showed a bright south pole $(\mathrm{N} / \mathrm{S}=0.87)$, which appeared negligibly dim above the tropopause (Jcont $-1.207 \mu \mathrm{m}: \mathrm{N} / \mathrm{S}=0.88$ ) and fading in the upper stratosphere $(\mathrm{J} 2-1.181 \mu \mathrm{m}: \mathrm{N} / \mathrm{S}=0.96)$. Three years later, the NSA has reversed, and is more pronounced at higher altitudes $(\mathrm{J} 2: \mathrm{N} / \mathrm{S}=1.16)$ than deeper in the troposphere (NB_1.04: N/S = 1.019). This demonstrates the slow motion and high inertia of the whole atmosphere: deeper layers respond more slowly to the change in solar insolation, as predicted by the models.

- Variations as a function of wavelength: the N/S ratios also appear to vary as a function of wavelength. In 2002, the NSA is definitely reversing $(2.12 \mu \mathrm{m}$ measurements are not counter-examples), yet in Table 2 the $\mathrm{N} / \mathrm{S}$ values are more contrasted at longer wavelengths than at $1 \mu \mathrm{m}$. In the $J$ band, the $\mathrm{N} / \mathrm{S}$ contrast varies by about \pm 0.2 (translating into variations in intensity of about $\pm 20 \%$ ), while it can reach 40 to $60 \%$ in the $K$ and $H$ bands. This may be due either to sensitivity to a particular size of aerosols and/or haze condensates, or merely to the actual altitude probed by the filters, revealing a more precise variation in the NSA as a function of altitude (see point above).

\section{Phase and diurnal effects on Titan}

Our images taken in the atmospheric filters contain a number of bright features. Some of them are due to normal solar phase effects, as can be seen on the western limb of Titan (to the left) in the 2002 images (Fig. 1). The brightness here is due to the 2.5 degree phase effect: the sunlit side of Titan is the brightest, because of diffusion and back-scattering by the atmospheric 
Table 3. E/W ratio characterizing the solar phase effect at various altitudes. Only relevant data are given here (surface images are not used). Some results are given in bold face (unexpected values indicative of a morning haze enhancement) or in italic (insecure values because error bars can change the interpretation); see text for details.

\begin{tabular}{|c|c|c|c|c|c|c|c|c|}
\hline Filter & $\begin{array}{c}\text { Alt limb } \\
\text { mini-maxi } \\
(\mathrm{km})\end{array}$ & $\begin{array}{l}\text { Oct. } 98 \\
\left(+0.5^{\circ}\right) \\
\end{array}$ & $\begin{array}{l}\text { Mar. 01 } \\
\left(+5.8^{\circ}\right) \\
\end{array}$ & $\begin{array}{l}\text { Dec. 01 } \\
\left(+0.3^{\circ}\right)\end{array}$ & $\begin{array}{c}\text { E/W ratio } \\
\text { Nov. } 02 \\
\left(-3^{\circ}\right) \\
\end{array}$ & $\begin{array}{l}\text { Jan. } 04 \\
\left(+0.8^{\circ}\right) \\
\end{array}$ & $\begin{array}{l}\text { Apr. 04 } \\
\left(+5.6^{\circ}\right) \\
\end{array}$ & $\begin{array}{l}\text { Jan. } 05 \\
\left(+0.2^{\circ}\right) \\
\end{array}$ \\
\hline NB_1.04 & $36-43$ & - & - & - & $0.907 \pm 0.011$ & - & - & - \\
\hline $\operatorname{Pa\gamma }$ & $5-21$ & - & - & $1.032 \pm 0.036$ & - & - & - & - \\
\hline $\mathrm{J} 2$ & $58-155$ & $\mathbf{0 . 8 9 7} \pm \mathbf{0 . 0 5 0}$ & $1.131 \pm 0.054$ & $0.976 \pm 0.023$ & $0.878 \pm 0.046$ & $1.008 \pm 0.084$ & - & \\
\hline Jcont & $43-65$ & - & $1.049 \pm 0.023$ & $0.982 \pm 0.035$ & - & $1.101 \pm 0.044$ & - & - \\
\hline NB_1.24 & $23-45$ & - & - & - & $0.854 \pm 0.006$ & - & - & - \\
\hline NB_1.64 & $45-322$ & - & - & - & $0.839 \pm 0.093$ & - & - & - \\
\hline $\mathrm{H} 2$ & $21-322$ & $0.970 \pm 0.082$ & - & - & $0.880 \pm 0.033$ & $1.000 \pm 0.349$ & - & - \\
\hline FeII & $45-280$ & - & - & $0.906 \pm 0.084$ & $0.851 \pm 0.058$ & $1.066 \pm 0.146$ & - & - \\
\hline NB_1.75 & $47-318$ & - & - & - & $0.876 \pm 0.009$ & - & - & - \\
\hline NB_2.12 & $17-20$ & - & - & - & $0.920 \pm 0.013$ & - & $1.328 \pm 0.060$ & $0.964 \pm 0.023$ \\
\hline $\mathrm{H} 2(1-0)$ & $17-24$ & - & - & $1.066 \pm 0.134$ & $0.868 \pm 0.061$ & $0.923 \pm 0.067$ & - & - \\
\hline IB_2.15 & $19-314$ & - & - & - & - & - & $1.093 \pm 0.061$ & $0.940 \pm 0.044$ \\
\hline $\operatorname{Br} \gamma$ & $40-359$ & - & - & - & $0.932 \pm 0.050$ & $1.033 \pm 0.140$ & - & - \\
\hline NB_2.17 & $40-359$ & - & - & - & $0.876 \pm 0.009$ & - & $1.329 \pm 0.111$ & $\mathbf{0 . 8 1 5} \pm \mathbf{0 . 0 3 7}$ \\
\hline
\end{tabular}

particles (haze in particular). The numerical values of the solar phase angle are given in Tables 1 and 3: if it is positive, then the solar phase effect is expected to appear on the eastern limb (this evening limb is on the right of all our north-up oriented images). When we find a brighter morning (i.e. west, on the left) limb in such conditions, we interpret this as a "dawn effect" (this diurnal stratospheric phenomenon was first described in Coustenis et al. 2001), characterizing a difference in atmospheric composition because of the nocturnal variations of the atmospheric conditions.

Table 3 and Fig. 4 summarize all the measurements of the $\mathrm{E} / \mathrm{W}$ contrast in atmosphere-probing images. As for the N/S contrasts, we consider profiles here of the intensity of the resolution elements chosen along the parallel where the E/W contrast is more pronounced: usually at the equator, except for the NACO 2005 images where the effect is more pronounced along the $30^{\circ} \mathrm{N}$ parallel because of the predominance of the bright northern limb. Error bars originate in the flux variations within three consecutive resolution elements on each limb, similar to what was described for the N/S ratios.

When the phase angle is large (e.g. March $2001\left(+5.8^{\circ}\right)$, November $2002\left(\right.$ about $\left.-3^{\circ}\right)$, and April $2004\left(+5.6^{\circ}\right)$ ), then the phase effect is obvious, with a $10-15 \%$ contrast between the eastern and western limbs, the brightness always on the expected side (E, W, and E, respectively).

In October 1998, December 2001, January 2004, and January 2005, the phase angle of our observations is small, and in some filters we do not readily detect the phase effect on the evening side; many of the contrast values are close to 1 within a $10 \%$ uncertainty. Narrow-band filters show that within error bars, the E and W limb fluxes are similar (many "insecure results" in December 01 and January 04 are in italics, underlining that the error bars are large enough to allow for a "phase-effect" solution). Yet in several cases (J2 and H2 in October 1998, J2 and FeII in December 2001, H2(1-0) in January 2004 and all NACO images in January 2005) error bars cannot account for the unusual aspect of the disk: the phase effect would have appeared faintly on the eastern limb (to the right) but the western limb is $3-19 \%$ brighter. We calculated these numbers from the profiles drawn in Fig. 3, which correspond to normalized intensity (1 being the brightest pixel of the image) calculated from one limb to another, along one parallel, the one where the effect is maximum returning the nominal value reported in Table 3 . With low positive values of the solar phase angle $\left(+0.2\right.$ to $\left.+0.5^{\circ}\right)$, the detection of such a brightness on the morning side could be attributed to a haze enhancement showing up in Titan's stratosphere at dawn (the 1998 PUEO data also allowed for such an interpretation in Coustenis et al. 2001).

We therefore find significant statistical evidence (with 7 firm detections and 8 tentative ones over the total of 16 images), on dates where it is possible (October 1998, March 2001, January 2004, and January 2005), of a bright morning stratospheric limb, which corroborates findings by Coustenis et al. (2001) based only on the 1998 PUEO images. Given the altitudes probed at the limb in these cases (see Table 3), this could be due to an enhancement of condensates during the Titan night (8 Earth days, though the super-rotation of Titan's atmosphere would lead to shorter nights for stratospheric clouds), manifesting itself in a morning haze enhancement phenomenon at stratospheric altitudes. Indeed, most of the images showing this phenomenon are mainly probing the stratosphere; between 40 and $311 \mathrm{~km}$ we can detect effects larger than $10 \%$, see Table 3. Exceptions are the $2.12 \mu \mathrm{m}$ filters, which mainly probe the troposphere: in this particular case we cannot deduce whether the detection corresponds to a faint nightly condensation occurring at these altitudes, or if it is a contribution of the larger phenomena in the stratosphere detected by the $2.12 \mu \mathrm{m}$ photons when they cross these layers before reaching the $\tau_{\text {eff }}=1$ level.

Such detections were also recently reported by other teams from independent observations with the Keck adaptive optics data (Brown et al. 2005; de Pater et al. 2005), probably because of the favorable observing conditions for such an effect during the Huygens probe descent in January 2005. Indeed, we find that the fainter the solar phase effect, the easier the detection of the "dawn effect". In January 2004 (phase $+0.8^{\circ}$ ), we had a firm $8 \%$ effect measurement, but all other images are insecure; furthermore, one image (Jcont) does show a regular phase effect. In December 2001 and October 1998 (phases of +0.3 and $+0.5^{\circ}$, respectively), we detected a $10 \%$ effect at most, with 3 detections and 4 uncertain solutions (but no regular phase effect). Finally, in January 2005 (phase $+0.2^{\circ}$ ), a $19 \%$ morning effect was detected at stratospheric levels, on all 3 images.

We therefore feel confident that this diurnal phenomenon is real and should be modeled in the near future in order to bring 

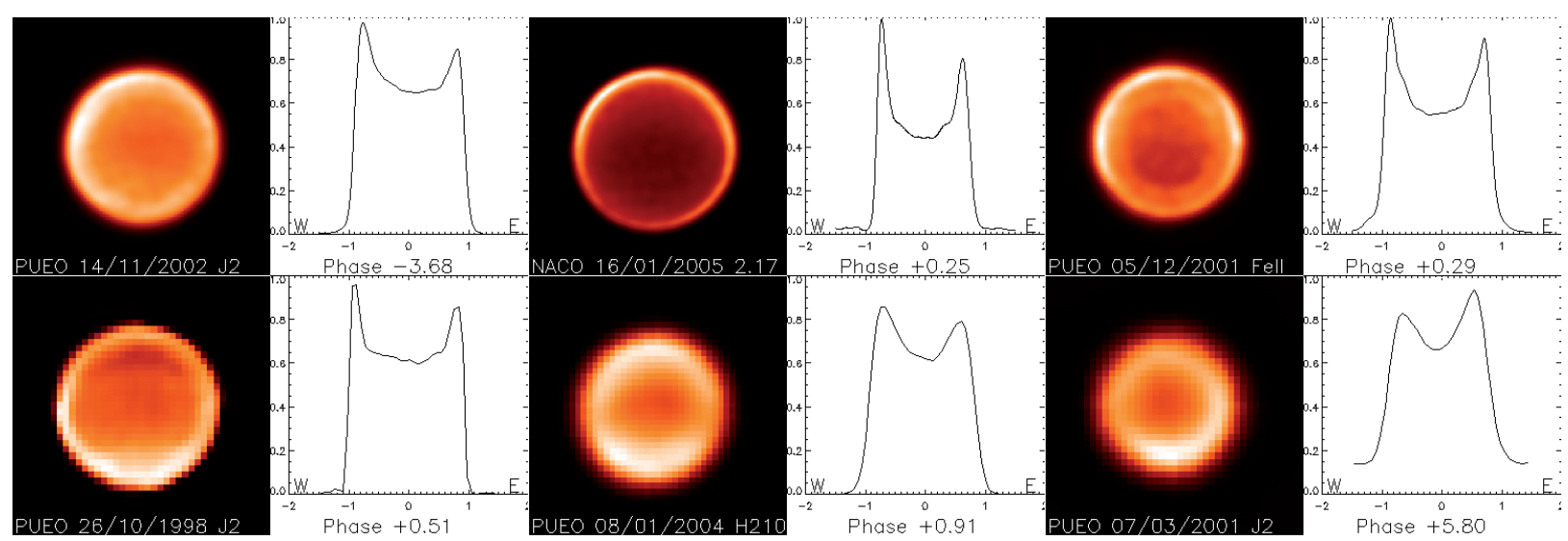

Fig. 3. Typical AO images showing the evolution of the east-west asymmetry on Titan as a function of solar phase angle, regardless of the actual date of observation (reported on the bottom of each image, along with the instrument used and the solar phase angle value). From upper left to bottom right, the solar phase angle increases, showing the reversal of the phase effect from the morning (west, left) to the evening (east, right) sides. The profiles drawn correspond to normalized intensity ( 1 being the brightest pixel of the image) from one limb to another, along one parallel, the one where the effect is maximum returning the nominal value reported in Table 3. At large phase angle values, the brightest limb corresponds to the sunlit one: western at negative phase angles (upper left), eastern at positive angles (bottom right). Yet at low phase angles (the four intermediate cases: upper centre, upper right, bottom left and bottom centre), the western limb (morning side) is always brighter than the eastern one (evening side), characteristic of a morning haze enhancement phenomenon related to a nocturnal condensation of stratospheric aerosols. See text for more details.

EWA Evolution

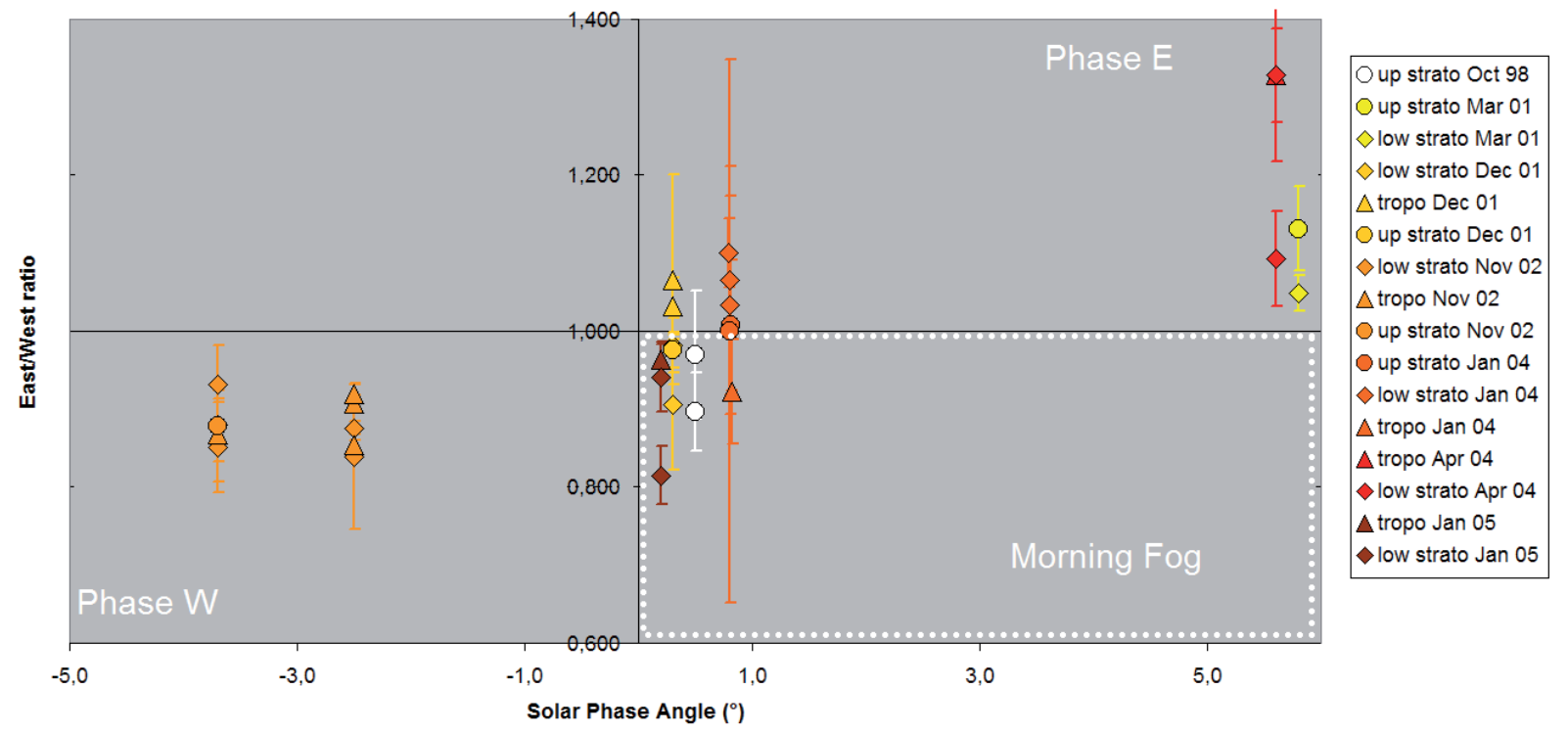

Fig. 4. Evolution of the EWA as a function of solar phase angle. Symbolism is similar to that used in Fig. 2: color indicates the year of the observation ( white $=1998$, yellow $=2001$, orange $=2002$, red $=2004$, brown $=2005$ ), and the symbol the altitude probed (triangles for the troposphere, diamonds for the tropopause, circles for the stratosphere). A datum in the bottom left corner of this plot corresponds to the regular phase effect detection expected for a west-lit diffusing object (phase effect on the morning limb), while in the upper right corner it corresponds to the regular phase effect on the east (evening) limb with a positive solar phase angle. A homogeneous diffusing atmosphere would display a linear curve reaching 1.0 at 0 phase angle, but this is not Titan's case. Seven occurrences show a bright western limb at low positive solar phase angle (the smaller the phase, the greater the stratospheric morning haze enhancement, reaching 19\% at most), in particular in October 98, December 01, and January 05. Our firm detections of diurnal effect are in the lower right quadrant.

new insights into Titan's atmospheric physics on a day-to-day basis.

\section{A southern bright revolving feature}

In addition to the features discussed in the previous sections, we have observed other discrete bright areas near Titan's south pole (sometimes atop a fine bright southern pole limb) in filters indicating that we are looking at the atmosphere at the limb (such as at $1.04,1.24$, and $2.12 \mu \mathrm{m}$ ). These features were first discernible in speckle images of Titan published by Gibbard et al. (1999). It has been extensively observed since 2001 in different filters (Fig. 5) and by different teams. It is particularly visible in $2.12 \mu \mathrm{m}$ images, where the southern limb appears after deconvolution as a group of three features: the southern polar feature (SPF) is bracketed by two elongated features, on the east and west sides, which we refer to here as EPF and WPF, respectively. Note the absence (or very rare presence) of bright features in the 


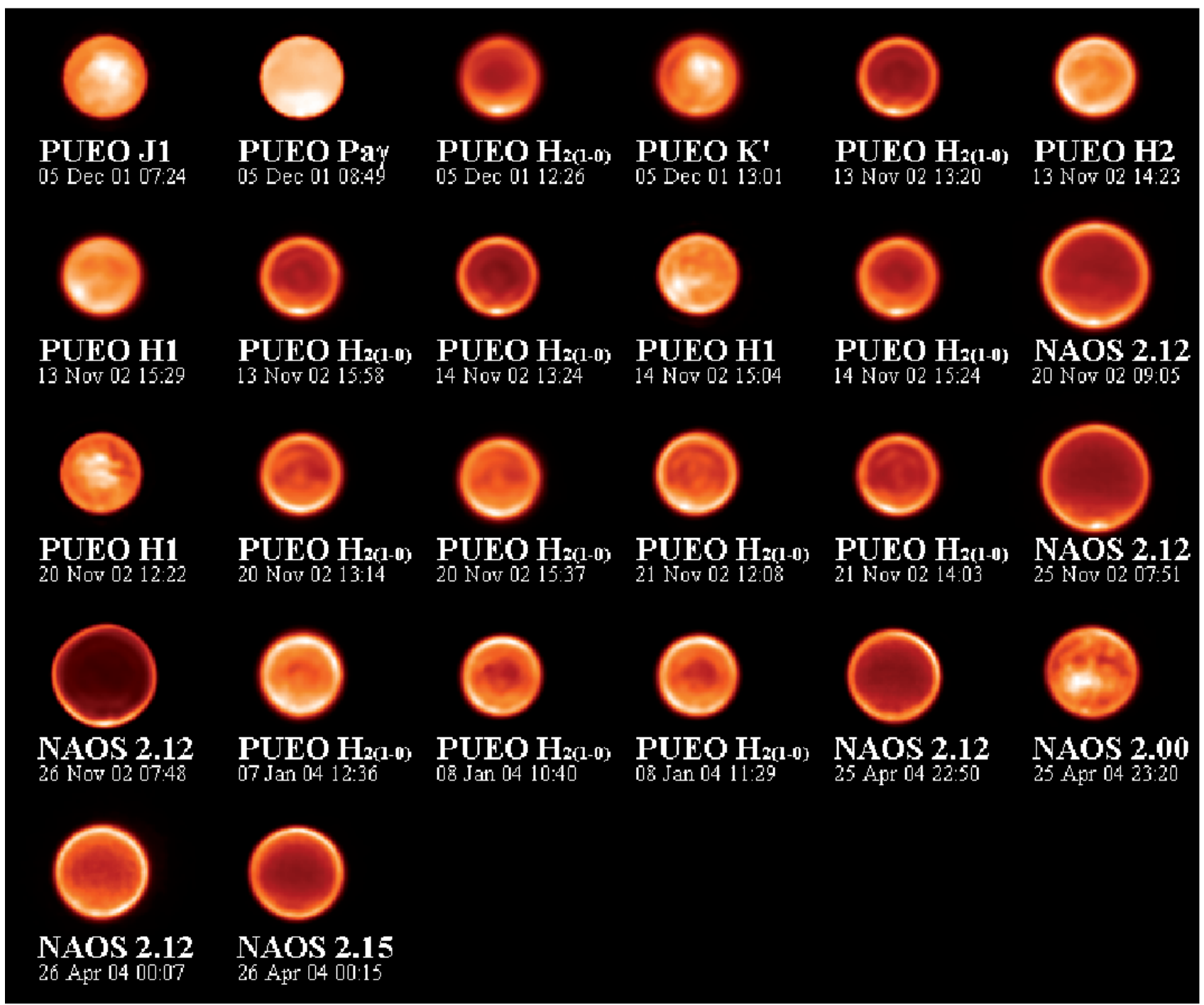

Fig. 5. The SPF is visible on all these images, sorted in chronological order. In 2002 we observed this feature several times during the night of 20 November with both PUEO and NACO, and repeated this scheme as often as possible in 2004 runs. The feature is brighter in 2001 than in more recent years, either because it was then enhanced by the additional brightness of the then southern limb of Titan and/or because it is now towards the end of its lifetime.

north, apart from the bright northern limb most certainly due to the NSA (the north Pole itself cannot be seen in most of our data, except in 1998).

\subsection{Description of the southern polar feature}

In this section we focus on the SPF evolving around and very near Titan's south pole especially seen in the $2.12 \mu \mathrm{m}$ images. This feature has been previously reported in observations from the Palomar, Keck, and Gemini taken:

- around $2 \mu \mathrm{m}$ : with a broad-band filter (also $K^{\prime} 0.3 \mu \mathrm{m}$ wide), on July 27-August 1, and 7-14 October 1998 (Gibbard et al. 1999, 2003);

- around $2.5 \mu \mathrm{m}$ : with a broad-band filter $\left(K^{\prime}, 1 \mu \mathrm{m}\right.$ wide $)$, on December 10 and 11, 2001 (Brown et al. 2002, 2005);

- around 2-2.25 $\mu \mathrm{m}$ with narrow-band filters (H2(1-0), NB2108) on 9, 18, 20, and 21 December 2001 (Roe et al. 2002).

- around $2 \mu \mathrm{m}$ (also $K^{\prime}$, but $0.3 \mu \mathrm{m}$ wide) in December 2001, September, November, and December 2002, and January 2003 (Bouchez et al. 2003, 2005);
It was interpreted by these authors as one of many transient methane clouds observed and located in the lower troposphere (5-20 km). For our part, we had also observed this phenomenon since 2001 and drawn attention to it (Coustenis et al. 2002, 2003; Gendron et al. 2004), marking its probable presence not in the lower, but more likely in the upper troposphere or in the stratosphere, thus noting its peculiar nature.

All of our SPF detections are summarized in Table 4 and Fig. 5. The phenomenon has been recently confirmed by Cassini observations at T0 and TA on 3 July and 26 October 2004, at much higher resolution (Porco et al. 2005). These authors now confirm the location of this cloud in the upper troposphere. A brief study was presented by Schaller et al. (2004) and Schaller et al. (2005) regarding the luminosity variation of the SPF within one year with a low-resolution telescope. Obviously the ground-based monitoring will be a valuable complement to the Cassini observations in order to constrain its nature and evolution.

We present here some inferences from our own survey of the SPF. Our images show this feature clearly in certain filters, in particular at $2.12 \mu \mathrm{m}$. Table 4 shows the position of the SPF 
Table 4. Observations of the SPF. Contrast values give the ratio of the SPF flux over the nadir flux supposed to be independent of the surface contribution (Non-applicable (NA) indications correspond to surface-probing filters).

\begin{tabular}{|c|c|c|c|c|c|}
\hline Instrument & Date/time (UT) & Filter & Altitude & Coordinates of S & Contrast \\
\hline PUEO & 05 Dec. 2001/07:24 & $\mathrm{J} 1$ & $0-58$ & $79 \pm 9 \mathrm{~S}, 157 \pm 122 \mathrm{~W}$ & $\overline{\mathrm{NA}}$ \\
\hline PUEO & 05 Dec. 2001/08:49 & $\mathrm{Pa} \gamma$ & $5-21$ & $84 \pm 12 \mathrm{~S}, 160 \pm 150 \mathrm{~W}$ & 1.14167 \\
\hline PUEO & 05 Dec. 2001/12:26 & $\mathrm{H} 2(1-0)$ & $17-24$ & $82 \pm 6 \mathrm{~S}, 156 \pm 70 \mathrm{~W}$ & 2.22147 \\
\hline PUEO & 05 Dec. 2001/13:01 & K' & 9-261 & $81 \pm 8 \mathrm{~S}, 225 \pm 69 \mathrm{~W}$ & NA \\
\hline PUEO & 13 Nov. $2002 / 13: 20$ & $\mathrm{H} 2(1-0)$ & $17-24$ & $81 \pm 9 \mathrm{~S}, 337 \pm 135 \mathrm{~W}$ & 2.42249 \\
\hline PUEO & 13 Nov. $2002 / 14: 23$ & $\mathrm{H} 2$ & $21-322$ & $87 \pm 13 \mathrm{~S}, 308 \pm 90 \mathrm{~W}$ & 2.12562 \\
\hline PUEO & 13 Nov. 2002/15:29 & H1 & 9-104 & $84 \pm 13 \mathrm{~S}, 54 \pm 50 \mathrm{~W}$ & NA \\
\hline PUEO & 13 Nov. 2002/15:58 & $\mathrm{H} 2(1-0)$ & $17-24$ & $83 \pm 7 \mathrm{~S}, 315 \pm 135 \mathrm{~W}$ & \\
\hline PUEO & 14 Nov. 2002/13:24 & $\mathrm{H} 2(1-0)$ & $17-24$ & $88 \pm 9$ S, 243 & 2.47067 \\
\hline PUEO & 14 Nov. 2002/15:04 & H1 & 9-104 & $86 \pm 12 \mathrm{~S}, 282 \pm 77 \mathrm{~W}$ & NA \\
\hline PUEO & 14 Nov. 2002/15:24 & $\mathrm{H} 2(1-0)$ & $17-24$ & $86 \pm 10 \mathrm{~S}, 282 \pm 102 \mathrm{~W}$ & 2.16502 \\
\hline NACO & 20 Nov. 2002/09:05 & 2.12 & $17-20$ & $80 \pm 4 \mathrm{~S}, 260 \pm 27 \mathrm{~W}$ & 2.25139 \\
\hline PUEO & 20 Nov. $2002 / 12: 22$ & H1 & 9-104 & $71 \pm 13 \mathrm{~S}, 249+/ 45 \mathrm{~W}$ & NA \\
\hline PUEO & 20 Nov. 2002/13:14 & $\mathrm{H} 2(1-0)$ & $17-24$ & $83 \pm 9 \mathrm{~S}, 237 \pm 123 \mathrm{~W}$ & 1.65806 \\
\hline PUEO & 20 Nov. $2002 / 15: 37$ & $\mathrm{H} 2(1-0)$ & $17-24$ & $81 \pm 8 \mathrm{~S}, 228 \pm 80 \mathrm{~W}$ & 1.63766 \\
\hline PUEO & 21 Nov. 2002/12:08 & $\mathrm{H} 2(1-0)$ & $17-24$ & $70 \pm 9 \mathrm{~S}, 209 \pm 27 \mathrm{~W}$ & 1.68432 \\
\hline PUEO & 21 Nov. 2002/14:03 & $\mathrm{H} 2(1-0)$ & $17-24$ & $72 \pm 11 \mathrm{~S}, 223 \pm 30 \mathrm{~W}$ & 1.99448 \\
\hline NACO & 25 Nov. 2002/07:51 & 2.12 & $17-20$ & $84 \pm 4 \mathrm{~S}, 312 \pm 42 \mathrm{~W}$ & 2.66154 \\
\hline NACO & 26 Nov. 2002/07:48 & 2.12 & $17-20$ & $84 \pm 5 \mathrm{~S}, 327 \pm 39 \mathrm{~W}$ & 5.23417 \\
\hline PUEO & 07 Jan. 2004/12:36 & $\mathrm{H} 2(1-0)$ & $17-24$ & $84 \pm 6 \mathrm{~S}, 112 \pm 110 \mathrm{~W}$ & 1.44520 \\
\hline PUEO & 08 Jan. 2004/10:40 & $\mathrm{H} 2(1-0)$ & $17-24$ & $80 \pm 10 \mathrm{~S}, 185 \pm 56 \mathrm{~W}$ & 1.81066 \\
\hline PUEO & 08 Jan. 2004/11:29 & $\mathrm{H} 2(1-0)$ & $17-24$ & $85 \pm 14$ S, $209 \pm 151 \mathrm{~W}$ & 1.76903 \\
\hline NACO & 25 Apr. $2004 / 22: 50$ & 2.12 & $17-20$ & $85 \pm 9 \mathrm{~S}, 185 \pm 46 \mathrm{~W}$ & 2.20384 \\
\hline $\mathrm{CO}$ & 25 Apr. 2004 & 2.00 & 9-158 & $88 \pm 4 \mathrm{~S}, 78 \pm 79 \mathrm{~W}$ & NA \\
\hline NACO & 26 Apr. 2004/00:07 & 2.12 & $17-20$ & $82 \pm 7 \mathrm{~S}, 150 \pm 30 \mathrm{~W}$ & 1.86792 \\
\hline NACO & 26 Apr. $2004 / 00: 15$ & 2.15 & $19-214$ & $81 \pm 7 \mathrm{~S}, 140 \pm 36 \mathrm{~W}$ & 2.31099 \\
\hline NACO & 16 Jan. 2005/03:58 & 2.12 & $17-20$ & $81 \pm 6 \mathrm{~S}, 355 \pm 90 \mathrm{~W}$ & 3.13592 \\
\hline
\end{tabular}

photocenter and its physical extent; the contrast value is given with respect to the centre of Titan's disk, when we can assume that the surface contribution is negligible, because we cannot compare the SPF luminosity with the southern limb or with any other limb, for too many features lie there that could bias the result: the NSA, the phase effects, the dawn haze enhancement, and so on. The shape of this feature does not seem to grow or flatten as a function of time within our resolution. Only its brightness seems to change, but we can correlate this more often than not with the appearance of the NSA; the brighter the southern limb, the brighter the SPF within it. Its average size corresponds to $2 \times 1$ elements of resolution for PUEO observations (pixel angular dimension is 0.0348 arcsec in this case) and to $3 \times 1.5$ for NAOS (pixel size is 0.01326 arcsec). This leads to an angular size of about $0.09 \times 0.05$ arcsec $( \pm 0.01)$ (according to Shannon's principle, the size of one resolution element must be twice the nominal angular size of the detector's pixels). This pertains to a projected surface reaching $700 \times 3000 \mathrm{~km}$, i.e. $4-5 \%$ of cloud cover (with respect only to the sunlit half of the satellite - i.e. $4.2 \times 10^{6} \mathrm{~km}^{2}-$ that we can see at a time).

We hereafter study the nature of this phenomenon observed in Titan's atmospheric images in view of the recent announcements of the detection of clouds over Titan's south pole (Brown et al. 2002; Roe et al. 2002a, 2005; Bouchez \& Brown 2005; Porco et al. 2005).

\subsubsection{Altitude of the SPF}

The detection of the SPF mainly occurs at $2.12 \mu \mathrm{m}$ in our images (at $2.108 \mu \mathrm{m}$ in Bouchez (2003) Palomar observations). We do have some hints that it is decipherable in other broadband filters $\left(J 1, K^{\prime}, H 2\right.$, or $H 1$ in particular), but such filters cover large spans of altitude within Titan's atmosphere, giving little indication of the exact position of the SPF. Since 2002, we have been using additional narrow-band filters in order to better restrain the SPF altitude. We had noticed that even though the SPF was bright and obvious at $2.12 \mu \mathrm{m}$, it was nowhere to be seen on $2.17 \mu \mathrm{m}$ images.

We will consider here for instance 2004 NACO data (some of which were published in Gendron et al. 2004), presented in Fig. 6. In the $2.00 \mu \mathrm{m}$ image, for instance, we note the presence of the SPF nearly on top of the south pole (lat $=85$ south $\pm 3^{\circ}$, long $=110 \mathrm{~W} \pm 20^{\circ}$ ). The surface contribution in this filter is predominant, but on the limb, we can probe down to the low troposphere $(9 \mathrm{~km})$. The SPF is usually particularly visible in $2.12 \mu \mathrm{m}$ images, where it is bracketed by the EPF and WPF features. In 2004, the predominance of the bright northern limb dampens the visibility of this feature, but it is still there. At $2.15 \mu \mathrm{m}$, the three features are still visible, but the SPF is Fading, sometimes around the "noise" level, and the WPF seems to be the brightest one. Finally, the central southern feature (SPF) and the two adjacent ones (EPF and WPF) have disappeared in images taken at $2.17 \mu \mathrm{m}$ (as also noted in Gendron et al. 2004).

According to our altitude estimates, the $2.17 \mu \mathrm{m}$ filter probes levels in the highest atmospheric regions. The EPF, WPF, and SPF are not visible above $80 \mathrm{~km}$. We probe deeper with IB_2.15, where the EPF and WPF appear, with the WPF slightly brighter than the EPF due to the phase effect. Deeper down the SPF appears. Further down in altitude, at 2.12 and then at $2.00 \mu \mathrm{m}$, only the SPF remains, very intense: the EPF and WPF are still there, detectable, but far less contrasted with respect to the SPF. We conclude that the EPF, WPF and SPF are atmospheric phenomena, since they do not appear in images of Titan's surface alone; they are located at different atmospheric levels, if we assume that the change in luminosity is due to both altitude and 


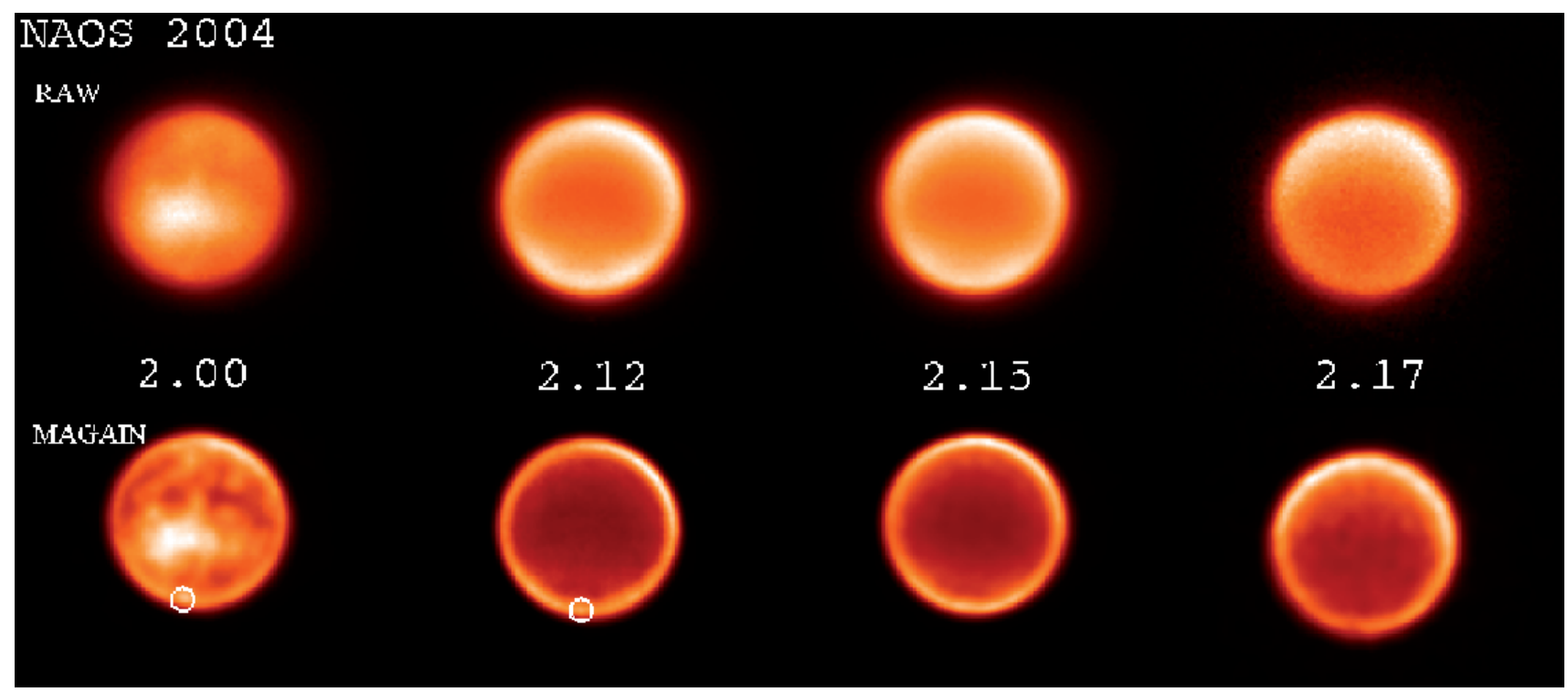

Fig. 6. Examples of detection and non-detection of the SPF in the $K$ band. The upper part displays the "raw" images before deconvolution, while the Magain-deconvolved ones lie on the bottom part. From left to right, images correspond to 2.00, 2.12, 2.15, and $2.17 \mu \mathrm{m} 2004$ NACO filters. Again, north is up and west-map to the left. When the SPF is present (see circles), it is bright on both raw and deconvolved images, while the two EPF and WPF companions only detach themselves clearly from the SPF on the deconvolved image.

phase effects and not solely to spectral features related to chemical composition.

For statistical purposes, we now consider our observations as a whole. We note that

- At 2.00 and $2.12 \mu \mathrm{m}$ (IB_2.00, H2(1-0), and NB_2.12), the SPF is clearly visible, along with its two EPF and WPF companions. The last, narrower filters probe down to $17-20 \mathrm{~km}$ (nominal value: $18 \mathrm{~km}$ ), implying that the SPF is certainly above $17 \mathrm{~km}$.

- On the NB_1.04, Pa $(1.09 \mu \mathrm{m})$ and IB_2.15 filters, the SPF is not always clearly visible, fading into the background along with both the EPF and WPF features. Depending on the observation, the SPF might be above 20 (IB_2.15, nominal: $50 \mathrm{~km}), 36$ (NB_1.04, nominal: $42 \mathrm{~km})$ or $50 \mathrm{~km}(\mathrm{~Pa} \gamma$, nominal: $53 \mathrm{~km})$.

- At $2.17 \mu \mathrm{m}$ (NB_2.17, Br $\gamma$ ), the SPF is not visible anymore, and the EPF and WPF merge into the southern limb. This implies that in the $40-311 \mathrm{~km}$ range probed by these filters there is no hint of the SPF. The preferential altitude probed here is about $83 \mathrm{~km}$, the upper limit for the SPF altitude.

As a conclusion, from the extreme values of the altitudes probed by these different filters, the SPF must be located in all probability above $17 \mathrm{~km}$ and below $40 \mathrm{~km}$, in which case it may well be a tropospheric condensation cloud; although its high altitude is a problem that modelers are still struggling to solve. Recent Cassini/ISS evaluations also locate this cloud in the upper troposphere (Porco et al. 2005).

Similarly, we conclude that the EPF and WPF lie above the altitude of the SPF, most probably above the tropopause; for instance, in 2004, the WPF was brighter at $2.15 \mu \mathrm{m}$ than at $2.12 \mu \mathrm{m}$, the latter probing deeper layers of the atmosphere. As we will explain below (Sect. 4.2), the EPF and WPF might be stratospheric haze layers looming above the south pole.

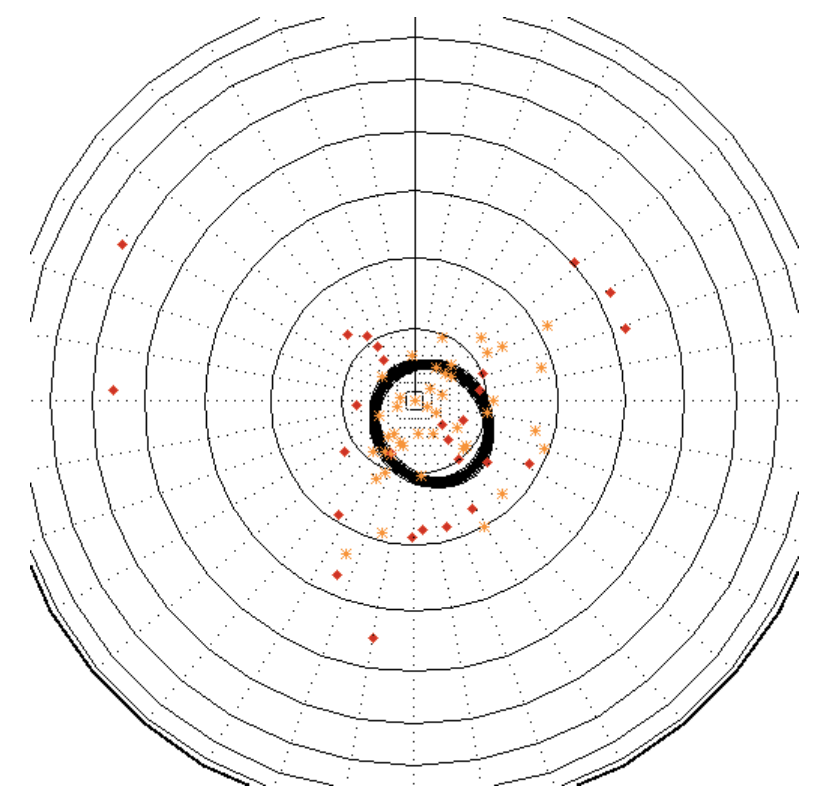

Fig. 7. Projections of the photocenter of the SPF on a spherical polar projection of Titan's southern hemisphere ( $\mathrm{LCM}=0^{\circ}$ meridian is up). Yellow stars correspond to our detections, as explained in Table 4. Red diamonds, extracted from Bouchez (2003), Bouchez \& Brown (2005) 1997-2003 data, are drawn for comparison only. The bold ellipse corresponds to the region of maximum detection, within which are confined $50 \%$ of the footprints shown here; $70 \%$ of them lay below the 80th parallel.

\subsubsection{Speed of the SPF}

We acquired several $2.12 \mu \mathrm{m}$ images within the same night on nearly all our 2002 and 2004 runs. With such series of data, we can compute speed values during the same night, or over one week at most. The velocity computation relies on the monitoring of the SPF position (given in Table 4 and plotted in Fig. 7): we assume the position of the SPF to be assimilated to the one of its brightest pixel. This is certainly a rough assumption, because 
it depends on the very shape of the real feature and on the conditions of illumination, but given the error bars due to the size of our resolution elements, we cannot be more accurate. We then consider the surrounding pixels that are $10 \%$ darker at most than the "photocenter" to be the actual extent of the SPF. This gives the error bars on the position as shown in Table 4. From these observations we find the SPF to often appear motionless within our error bars. From one night to another, we detected a displacement from which we compute an average velocity of $3 \pm 2 \mathrm{~m} / \mathrm{s}$. This value is compatible with all wind speed values reported from observations of the upper troposphere of Titan $(0.5 \pm 3 \mathrm{~m} / \mathrm{s}$ by VIMS - Momary et al. (2004), $34 \pm 13 \mathrm{~m} / \mathrm{s}$ at most by ISS Porco et al. (2005), and $25 \mathrm{~m} / \mathrm{s}$ at $30 \mathrm{~km}$ - Tomasko et al. (2005)) and models of raw winds in the troposphere (Hourdin et al. 1995; Tokano et al. 2001; Rannou et al. 2004). From this velocity computation we can confirm the 4.1.1 conclusion that the SPF more probably lies in the upper troposphere if we suppose that both the clouds and their constituents are driven by the average winds. (Sometimes the apparent speed of one cloud is not related to the speed of its particles, as for instance the case of orographic clouds.)

Nonetheless our computations show in some cases an apparent speed that can reach about $120 \mathrm{~m} / \mathrm{s}\left(167_{-96}^{+230} \mathrm{~m} / \mathrm{s}\right.$ from the positions measured on the 13 November 2002 $\mathrm{H} 2$ and $\mathrm{H} 1$ images; a similar case occurs in the measurements on the 25 April 2004 IB_2.00 and NB_2.12 images returning a $122_{-49}^{+81} \mathrm{~m} / \mathrm{s}$ velocity. Such high speed motions would correspond to upper stratospheric winds (reaching at least $75 \mathrm{~m} / \mathrm{s}$ at $200 \mathrm{~km}$ - Luz et al. 2005 - or $30 \mathrm{~m} / \mathrm{s}$ at $50 \mathrm{~km}$ - Tomasko et al. 2005). A joint operation between the JPL, the JIVE/VLBI, and Cassini-Huygens/DWE (Bird et al. 2005) teams measured prograde wind speeds of about $120 \mathrm{~m} / \mathrm{s}$ at $120 \mathrm{~km}$ - http://wWW.esa.int/SPECIALS/ Cassini-Huygens/SEMA8SXEM4E_Q.html. We cannot explain why the trajectory of the SPF would be so erratic, unless it is a sign of shape changes. Indeed, as confirmed by VIMS (Brown et al. 2005) and ISS (Porco et al. 2005) observations at T0 and TA: the SPF looks like a large ring of thin clouds or haze that changes in shape with little bright clouds trapped within it, appearing and disappearing randomly, and recently disintegrating, something we cannot resolve with Earth-based AO systems. Thus, our high speed computations are only an indication of such deforming of the SPF. As a conclusion, the regular most probable averaged velocity value we find is $3 \pm 2 \mathrm{~m} / \mathrm{s}$. With such a value, we cannot ascertain that the SPF is really revolving around the south pole of Titan, we can only suggest that if it was moving, it was following a slow prograde motion at the times of our observations.

\subsection{The case of the EPF and WPF companions}

In all images, the SPF feature is paired with two unmoving companions on each side of the limb. They are neither moving nor changing in shape, yet one is always longer than the other; more precisely, the one on the sunlit side is usually longer: for instance, on the $20022.12 \mu \mathrm{m}$ image in Fig. 1, the solar phase effect is on the western limb, and the WPF companion is spanning farther out on the limb. We believe the gap between the bright SPF and these two companions is a deconvolution artifact, because it is less than one resolution-element wide, and the Shannon principle requires it to be at least twice as large to be physically valid.

Many atmospheric models (Rannou et al. 2002, 2004 for instance) foresee two stratospheric hoods of haze swept away from the Equator by the atmosphere dynamics. The two companions may be a deformed appearance of such a hood; and there is evidence of a strange serpentine feature at latitudes below the 60th parallel, also described as an "equatorial cirrus" (Roe et al. 2002a) that might represent the upper limit of this hood. We can therefore suppose that this hood is brighter on the solar-enlightened limb and is uniformly encompassing the south pole southward from the 60th parallel. Within this hood lies the SPF. After deconvolution, the hood would be deformed: the ringing effect only enhances the portion of the hood overlapping the disk's limb, and there is only a small indication of its northern extent, near the southern third of the disk. Furthermore, a gap can be created by the deconvolution around the SPF, artificially darkening the limb and creating this "two companions" appearance. This led us to omit all the 2.12 images for the NSA evolution quantification, because there are too many features and artifacts mixing with each other to allow for a correct reading of the intensity of the southern limb.

Finally, we have only three choices left: the companions are either real atmospheric features (clouds?), a deconvolution artifact, or a deformed signature for a hypothetical haze polar hood.

\section{Conclusions and discussion}

We have studied Titan's atmosphere with adaptive optics in the past 8 Years, and we report on results pertaining to atmospheric seasonal, Diurnal, and meteorological phenomena.

1. Titan's north-south asymmetry has reversed from the situation where in 1992 HST found the south pole to be brighter than the northern (Caldwell et al. 1992) in the near-infrared. This reversal is noted since 2001 in the data presented here (but was found to exist earlier from other studies in the visible). Our data show that this NSA reversal occurs first at higher altitudes (the ones more sensitive to solar input) and then propagates to lower levels. By 2004, the NSA is completely inverted at all wavelengths between 1 and $2 \mu \mathrm{m}$.

2. The diurnal effects ("morning fog" or more precisely "morning haze enhancement") first described in Coustenis et al. (2001) are confirmed in our observations (in particular in the 2005 VLT data). Phase effects appear on the limb closer to the sub-solar point, as expected, with the corresponding limb up to $15 \%$ brighter than the opposite one in the case of a large $\left(3^{\circ}\right)$ solar phase angle. Other images, at low solar phase angles with a faint phase effect on the evening side, allow us to detect a stronger diurnal effect on the morning terminator: about $4-6 \%$ in the troposphere and $19 \%$ in the stratosphere. We have made a statistically convincing case for the presence of such a phenomenon on Titan.

3. There is a large bright feature revolving around the south pole of Titan (mainly within the $80^{\circ}$ parallel). Our data indicate that it is located between 17 and $83 \mathrm{~km}$ of altitude, more probably (as expected for a condensation cloud) in the upper troposphere $(17-40 \mathrm{~km})$. We find from the footprints of our observations that the trajectory associated with the movement of this feature is erratic: most velocities indicate $3 \mathrm{~m} / \mathrm{s}$; but in some cases, this speed is significantly higher, which is compatible with a fast deformation of the feature as witnessed in Cassini images.

\subsection{The north-south asymmetry}

The NSA of Titan has been recorded since the Voyager encounters in the late 70's. At that time, Titan's season was just after 
the vernal equinox, with the southern hemisphere brighter by about $20 \%$ than the northern in the visible (Smith et al. 1981, 1982; Letourneur \& Coustenis 1993). This tendency reversed in 1990 in the blue $(440 \mathrm{~nm})$ and yellow $(550 \mathrm{~nm})$ wavelengths, with the northern hemisphere about $10 \%$ brighter than the southern (HST measurements by Caldwell et al. 1992). Indication of a further reversal since 1997, in the visible again, from HST images was found (Lorenz et al. 1999, 2001), with the "smile" disappearing at $889 \mathrm{~nm}$, although still visible at $953 \mathrm{~nm}$ and beyond.

These albedo changes are interpreted as seasonal variations (Sromovsky et al. 1981; Lockwood et al. 1986) related to the haze properties (such as the albedo increase in the haze particles with wavelength, McKay et al. 1989; Griffith et al. 1991). It seems that the NSA reversal settles in over a 5-year period (Lorentz et al. 2001 or Roe et al. 2002), which is faster than the seven-year period between equinox and solstice on Titan. Sromovsky et al. (1981) find a phase lag for the NSA of about 90 degrees relative to the solar.

Several mechanisms were proposed to explain the NSA (see Roos-Serote 2004 for a review), but dynamics seem to be the most plausible answer: Hutzell et al. (1993) showed that microphysics alone (via changes of chemical composition or in products concentration) cannot fully explain the NSA reversal. The spatial repartition of the haze, coupled with dynamical effects (Hutzell et al. 1995), can reproduce the changes in appearance of Titan's atmosphere as seasons go by. Recent GCM models can even reproduce the 5-year settling period (see in particular Sect. 5 of Luz et al. 2003).

Previous measurements have indicated that during the Cassini/Huygens mission the NSA would be stable. From our data, and because we have altitude-discriminating information in our narrow-band filters, a more complicated pattern appears. We have followed the evolution of the NSA in the past decade, and done so more efficiently in the past 5 years. We find it to be more pronounced at some wavelengths probing the higher atmosphere and to be slowly affecting the lower levels and down to the troposphere at the current era.

Such information is crucial to the understanding of the atmosphere dynamics, and can be used to constrain current seasonal/photochemical/dynamical models, since it quantifies the spatial and temporal propagation of the NSA.

\subsection{The diurnal effects}

Our images show a significant number of cases where a bright morning limb in the stratosphere is seen when the phase effect is expected on the evening side. These cases were observed principally in filters probing atmospheric levels between 20 and $300 \mathrm{~km}$ (but mainly the stratosphere, Table 3).

Microphysical models of Titan's haze (e.g. Rannou et al. 2003) predict the condensation of methane by-products (such as ethane and acetylene as the most abundant ones) somewhere between 70 and $90 \mathrm{~km}$ of altitude in the polar regions (Coustenis et al. 2001). Thus, the observed morning/evening asymmetry that we have found in our data since 1998 could be explained by a diurnal condensation cycle of the condensable species. Since on Titan one night lasts about 8 terrestrial days, even considering stratospheric winds of about $100 \mathrm{~m} / \mathrm{s}$ (Kostiuk et al. 2001; Luz et al. 2005) that would shorten the length of the night to 1 Earth day for the particles at that altitude, there is enough time for the condensation to occur at the low temperatures prevailing on the night side. As a matter of fact, if condensation nuclei are present, only a few $10^{-5} \mathrm{~s}$ are needed to begin the condensation (Pruppacher \& Klett 1978). This nocturnal condensation then manifests itself by a brighter cold morning limb with respect to the darker warmer evening side.

The general circulation model developed for the atmosphere of Titan at the Institute Pierre-Simon Laplace (Hourdin et al. 1995, 2004; Rannou et al. 2002, 2004) can give a cloud production from temperature variations reaching 0.1 to $0.2{ }^{\circ} \mathrm{K}$ in regions of supersaturation, and we are investigating the temperature variation we can expect on Titan between the day and night hemispheres.

\subsection{Investigating the nature of the SPF}

The SPF has been currently observed many times from the ground and space, including by Cassini (Porco et al. 2005; Brown et al. 2005). Its very motion is incompatible with a surface-related feature, although it might be some orographic cloud or another surface-related phenomenon, like cryovolcanism-induced plumes (Tobie et al. 2005). It has been observed for 5-6 years, close to the duration of one season on Titan. It was first noticed in 1999 by Gibbard et al. (1999), then it was confirmed in 2001 from Palomar (Bouchez \& Brown 2005) and PUEO and NACO observations (this work). At the beginning of 2005, the SPF completely disappeared from view in both Earth-based (Griffith et al. 2005; Hirtzig et al. 2006) and Cassini data (Porco et al. 2005). This indication of a specific lifetime for the whole system now has to be taken into account by Titan's atmosphere modelers.

An important point of our investigation is to constrain the nature of the SPF we detect. The SPF could be the spatial average of a large system of clouds, whose transient appearances is ruled both by the condensation, precipitation, or evaporation processes and by the very motions, vertical, and/or lateral of the atmosphere. This could explain why the SPF deforms so quickly from time to time: the first individual cloud disappears while another one is created at another place, still within the larger atmospheric system. We then have to describe both the clouds and the whole atmospheric region.

The SPF is often detected poleward of the 70th parallel at an altitude of $18-40 \mathrm{~km}$ in the upper troposphere. This is confirmed by the average $3 \mathrm{~m} / \mathrm{s}$ speed value we have computed for the feature's apparent movement, which is within the same order of magnitude as the VIMS results for tropospheric winds (Momary et al. 2004). Yet our computation does not give the speed of the particles carried by the wind, but only the apparent speed of the bright feature atop Titan's south pole, which can be lower than the wind speed. The difference can be illustrated by the typical example of an orographic cloud, whose moist airmass is being carried away by the wind, although it seems not to move.

The extent of the SPF in our image is closely related to the spatial resolution of our instruments, and it reaches $700 \times$ $3000 \mathrm{~km}$. It is difficult to have a better constraint on the size because of three main drawbacks: (1) the actual spatial resolution of our images, (2) observational effects (we observe the SPF close to the limb), and (3) deconvolution effects (the position of the SPF on the edge of the disk changes slightly because of the Gibbs ring). Other detections from Earth (Brown et al. 2005) return the same order of size, since we are mainly limited by the diameter of our instruments. The ISS/Cassini images show that the whole system is more probably some $1000 \mathrm{~km}$ wide, weakly centered on the south pole (Porco et al. 2005). It contains a number of small $100-\mathrm{km}$ sized clouds that appear and disappear randomly in this region. 
The overall brightness of the SPF (about 100 to $300 \%$ brighter than the surrounding areas) can be explained by two hypotheses: the individual clouds can be large-sized (with respect to our spatial resolution) or/and the individual clouds can be highly reflective. The Cassini images confirm the latter explanation, but do not rule out the former one, because large changes in luminosity were observed in past years (Schaller et al. 2005), due to the meteorological activity, thereby perhaps inducing a larger number of small bright clouds, or maybe increasing the average size of the individual clouds.

Each individual cloud is a very bright feature, probably a methane/ethane cloud (driven by convection, see Roe et al. 2005) or a thunderstorm, characterized by a short lifespan (about 2-3 days up to one week). Convection can only work in the lower part of the tropopause, forcing the altitude of the SPF to be less than $30 \mathrm{~km}$ (which is not certain). Its reflectivity might be due to a particular signature of its components: aerosols? dust? methane/ethane snowflakes/droplets?. We can only guess its origin; if the "supersaturation of methane" (Courtin et al. 1995; Samuelson et al. 1997) hypothesis is confirmed, then the creation of a cloud requires a large amount of CCN (cloud condensation nuclei) to account for the large lifespan of the cloud; the creation of such CCN may involve cryovolcanism (Lorenz 1996, 2002) ejecting dust particles into the lower troposphere, or aerosols brought from the upper stratosphere down into the troposphere by descending motions (as suggested by models: Hourdin et al. 2004; Rannou et al. 2004). On the other hand, the formation of clouds would be easier if there were (locally or globally) no supersaturation, but then the formation of clouds should not be limited only to the south pole of Titan. Another explanation has also been invoked: that this phenomenon is a cloud produced from the evaporation of methane ice at the south pole (Lorenz 1996, 2002; Roe et al. 2005; Griffith et al. 2005).

The large phenomenon entrapping the individual clouds seems circular, as seen by Cassini, probably revolving around the south pole (our computations lead to a feeble but not nil prograde motion). The GCM model (Hourdin et al. 1995 and updates) may give insights into how such a structure may be produced in the troposphere of the summer hemisphere. While the dominant circulation pattern seen in the model is a large stratospheric Hadley cell with ascending motions in the summer hemisphere and subsiding motions above the winter pole, a small stratospheric Hadley cell persists above the summer pole (see e.g. Fig. 10 of Hourdin et al. 2004). This cell may explain why an enhancement of ethylene has been observed late in spring in the southern hemisphere (Roe et al. 2004). It keeps high aerosol concentrations in this region, and induces downward motions of trace compounds such as ethane, acetylene, and hydrogen cyanide, explaining why condensation is obtained in the GCM over the summer pole, above the tropopause (Hourdin et al. 2004). These high aerosol particle densities provide condensation nuclei, wetted by nitriles and other trace species, on which methane condensation may occur if transport mechanisms are able to bring high abundances of methane from the surface into this region. A new version of the GCM currently under development couples cloud microphysics with the dynamics and hazes, and first results show that, indeed, in the summer hemisphere, circulation in the troposphere transport methane-rich air masses from the mid-latitude surface into the polar troposphere, producing clouds that share many similarities with the observed SPF (Rannou et al. 2005) and with the Cassini images.

The feature we observe may also be connected with large-scale planetary waves. Simulations of such waves with a shallow-water model (Luz \& Hourdin 2003) indicate they play a role in mixing tracers, and could be involved in cloud formation. The difficulty remains, however, that the south polar feature is located close to the summer pole, whereas wave activity should be stronger in the winter hemisphere. Another possibility (Flasar 1998) would be the disruption of the polar vortex by planetary waves, as the Earth's stratosphere during winter (Juckes \& McIntyre 1987). Again, this remains a speculative but promising hypothesis, since modeling predicts significant wave activity to occur close to the summer solstice (Luz et al. 2003).

It may require more long-term observations and follow-up of this occurrence before we fully comprehend its nature.

Acknowledgements. We thank Amélie Gasson for her assistance. Many thanks also to Panagiotis Lavvas for his advice and fruitful discussions. Some of the data presented here are based on observations collected at the European southern Observatory, Chile (ESO proposals 70.C-0588, 73.C-0221 and 74.E-0747 for guarantee time with NACO). A. Negrão is supported by the FCT PhD scholarship SFRH/BD/8006/2002. D. Luz acknowledges financial support from Fundação para a Ciência e a Tecnologia, Portugal (fellowship PRAXIS XXI/BPD/3630/2000 and project POCTI/CTE-AST/57655/2004). Mathieu Hirtzig was a Visiting Astronomer from 30 December 2003 to 8 January 2004 at the Canada-France-Hawaii Telescope operated by the National Research Council of Canada, the Centre National de la Recherche Scientifique de France, and the University of Hawaii.

\section{References}

Bézard, B., Coustenis, A., \& McKay, C. P. 1995, Icarus, 113, 267

Blanc, M., Bolton, S., Bradley, J., et al. 2002, Space Sci. Rev., 104, 253

Bouchez, A. H. 2003, Ph.D. Thesis

Bouchez, A. H., \& Brown, M. E. 2005, ApJ, 618, 53

Bouchez, A. H. Brown, M. E., Neyman, C. R., et al. 2005, submitted, Groundbased visible adaptive optics observations of Titan's atmosphere and surface

Boudon, V., Rey, M., \& Loëte, M. 2006, J. Quant. Spectrosc. Radiat. Trans., 98, 394

Bratsolis, M., \& Sigelle, M. 2001, A\&A, 375, 1120

Brown, M. E. 2005, The seasonal hydrological cycle on Titan, in Proceedings of the Conference Titan after the Huygens and First Cassini Encounters, Crete, Greece, 30 May-3 June 2005:

wwW. lpl . arizona.edu/titanconference/index.html

Brown, M. E., Bouchez, A. H., \& Griffith, C. A. 2002, Nature, 420, 6917, 7995

Brown, R. H., Baines, K. H., Bellucci, G., et al. 2005, Cassini VIMS at Saturn: The First 6 Months, 36th Annual Lunar and Planetary Science Conference, March 14-18, 2005, in League City, Texas, \#1166

Caldwell, J., Cunningham, C. C., Anthony, D., et al. 1992, Icarus, 97, 1

Chanover, N. J., Anderson, C. M., McKay, C. P., et al. 2003, Icarus, 163, 1, 150

Clarke, J. T., Gérard, J.-C., Grodent, D., et al. 2005, Nature, 433, 717

Combes, M., Vapillon, L., Gendron, E., et al. 1997, Icarus, 129, 2, 482

Conan, J. M. Fusco, T., Mugnier, L., et al. 1998, Deconvolution of adaptive optics images with imprecise knowledge of the point spread function: Results on astronomical objects, in Astronomy with Adaptive Optics: Present Results and Future Programs, ESO/OSA Workshop, Sept. 1998, Sonthofen, Germany

Courtin, R., Gautier, D., \& McKay, C. P. 1995, Icarus, 114, 144

Coustenis, A., Lellouch, E., Maillard, J. P., \& McKay, C. P. 1995, Icarus, 118, 87

Coustenis, A., \& Bézard, B. 1995, Icarus, 115, 126

Coustenis, A., Gendron, E., Lai, O., et al. 2001, Icarus, 154, 501

Coustenis, A., Hirtzig, M., Lai, O., et al. 2002, BAAS, 34, 881

Coustenis, A., Hirtzig, M., Lai, O., et al. 2003, New adaptive optics images of Titan with the CFHT/PUEO: disk-resolved description of atmospheric and surface features, 28th General Assembly of the European Geophysical Society, Nice, 7-11 April

Coustenis, A., Hirtzig, M., Gendron, E., et al. 2005, Icarus, 177, 89

Coustenis, A., Negrão, A., Salama, A., et al. 2006, Icarus, 180, 176

de Pater, I., Adamkovics, M., Gibbard, S., et al. 2005, Keck observations of Titan during probe entry and during the days following touchdown. EUROPLANET N3 activity Kick-off meeting, Graz, 8 March, and http://europlanet.oeaw.ac.at/ N3_Meeting_09032005.html

Flasar, F. M., Samuelson, R. E., \& Conrath, B. J. 1981, Nature, 292, 693

Flasar, F. M. 1998, PSS, 46, 1109 
Gendron, E., Coustenis, A., Drossart, P., et al. 2004, A\&A, 417, L21

Gibbard, S. G., Macintosh, B. A., Gavel, D., et al. 1999, Icarus, 139, 189 33, 1403

Gibbard, S. G., Macintosh, B. A., Max, C. E., et al. 2001, BAAS, 33, 1403

Griffith, C. A., Owen, T., \& Wagener, R. 1991, Icarus, 93, 362

Griffith, C. A., Owen, T., Miller, G. A., \& Geballe, T. 1998, Nature, 395, 575

Griffith, C. A. Hall, J. L., \& Geballe, T. R. 2000, Science, 290, 509

Griffith, C. A. Owen, T., Geballe, T. R., et al. 2003, Science, 300, 5619, 628

Griffith, C. A., Pentadeo, P., Baines, K., et al. 2005, Science, 310, 474

Hilico, J. C., Champion, J. P., Toumi, S., et al. 1994, J. Mol. Spectrosc., 168, 455

Hirtzig, M., Coustenis, A., Gendron, E., et al. 2004, 36th Annual DPS Meeting, Louisville, Kentucky (USA), 7-12 Novembre, BAAS, 36, 1120

Hirtzig, M., Coustenis, A., Gendron, E., et al. 2006, JGR, submitted

Hourdin, F., Talagrand, O., Sadourny, R., et al. 1995, Icarus, 117, 358

Hourdin, F., Lebonnois, S., Luz, D., \& Rannou, P. 2004, J. Geophys. Res., 109, 12005

Hutzell, W. T., McKay, C. P., \& Toon, W. B. 1993, Icarus, 105, 162

Hutzell, W. T., McKay, C. P., Toon, W. B., \& Hourdin, F. 1996, Icarus, 119, 112

Irwin, P. G. J., Sihra, K., Bowles, N., et al. 2005, Icarus, 176, 255

Irwin, P. G. J., Sromovsky, L. A., Strong, E. K., et al. 2006, Icarus, in press

Juckes, M. N., \& McIntyre, M. E. 1987, Nature, 328, 590

Kostiuk, T., Fast, K. E., Livengood, T. A., et al. 2001, Geophys. Res. Lett., 28, 2361

Lemmon, M. T., Karkoschka, E., \& Tomasko, M. 1993, Icarus, 103, 329

Lemmon, M. T., Karkoschka, E., \& Tomasko, M. 1995, Icarus, 113, 1, 27

Letourneur, B., \& Coustenis, A. 1993, Space Sci., 41, 593

Lockwood, G. W., Lutz, B. L., Thompson, D. T., \& Bus, E. A. 1986, ApJ, 303, 511

Lorenz, R. D. 1996, Planet. Space Sci., 44, 1021

Lorenz, R. D. 2002, Icarus, 156, 176

Lorenz, R. D., Smith, P. H., Lemmon, M. T., \& Karkoschka, E. 1997, Icarus, 127,173

Lorenz, R. D., Lemmon, M. T., Smith, P. H., \& Lockwood, G. W. 1999, Icarus, 142,391

Lorenz, R. D. E. F., Young, \& Lemmon, M. T. 2001, Geo. Res. Lett., 28, 23, 4453

Luz, D., \& Hourdin, F. 2003, Icarus, 166, 238

Luz, D., Hourdin, F., Rannou, P., \& Lebonnois, S. 2003, Icarus, 166, 343

Luz, D., Civeit, T., Courtin, R., et al. 2005, Icarus, 179, 497

McKay, C. P., Pollack, J. B., \& Courtin, R. 1989, Icarus, 80, 23

Meier, R., Smith, B. A., Owen, T. C., \& Terrile, R. J. 2000, Icarus, 145, 462

Momary, T. W., Baines, K. H., Buratti, B. J., et al. 2004, Not So Titanic Winds: Cassini/VIMS Observations of Cloud Features in the southern Hemisphere of Titan, 36th DPS meeting, \#06.10

Negrão, A., Coustenis, A., Lellouch, et al. 2006, Icarus, in press

Neubauer, F. M., Gurnett, D. A., Scudder, J. D., \& Hartle, R. E. 1984, Titan's magnetospheric interaction, in "Saturn", ed. T. Gehrels, \& M. S. Matthews (Univ. Arizona Press), 760

Pollack, J. B., \& McKay, C. P. 1985, J. Atmos. Sci., 42, 245
Porco, C. C., Baker, E., Barbara, J., et al. 2005, Nature, 434, 159

Pruppacher, H. R., \& Klett, J. D. 1978, Microphysics of clouds and precipitation Rages, K., \& Pollack, J. B. 1980, Icarus, 41, 119

Rages, K., Pollack, J. B., \& Smith, P. H. 1983, J. Geophys. Res., 88, 8721

Rannou, P., Cabane, M., Chassefiere, E., et al. 1995, Icarus, 118, 355

Rannou, P., Hourdin, F., \& McKay, C. P. 2002, Nature, 418, 853

Rannou, P., McKay, C. P., \& Lorenz, R. D. 2003, Planet. Space Sci., 51, 963

Rannou, P., Hourdin, F., McKay, C. P., \& Luz, D. 2004, Icarus, 170, 443

Rannou, P., Montmessin, F., Hourdin, F., \& Lebonnois, S. 2005, Geophys. Res. Abstracts, 7, 04185

Rigaut, F., Salmon, D., Arsenault, R., et al. 1998, Performance of the CanadaFrance-Hawaii Telescope adaptive optics bonnette, Pub. Astron. Soc. Pac. $110,744,152$

Ringrose, T. J., Towner, M. C., \& Zarnecki, J. C. 2003, Icarus, 163, 78

Roe, H. G., de Pater, I., Macintosh, B. A., \& McKay, C. P. 2002a, ApJ, 581, 2, 1399

Roe, H. G., de Pater, I., Macintosh, B. A., et al. 2002b, Icarus, 157, 1, 254

Roe, H. G., de Pater, I., \& McKay, C. P. 2004, Icarus, 169, 440

Roe, H. G., Bouchez, A. H., Trujillo, C. A., et al. 2005, ApJ, 618, 49

Saint-Pé, O., Combes, M., Rigaut, F., et al. 1993, Icarus, 105, 263

Samuelson, R. E., Nath, N. R., \& Borysow, A. 1985, P\&SS, 45, 959

Schaller, E. L., Brown, M. E., Bouchez, A. H., et al. 2004, BAAS, 36, 1076

Schaller, E. L., Brown, M. E., Bouchez, A. H., \& Trujillo, C. A. 2005, Cloud activity on Titan during the Cassini mission, 36th Annual Lunar and Planetary Science Conference, March 14-18, 2005 in Leagu City, Texas, \#1989

Sihra, K. 1998, Ph.D. Thesis, University of Oxford

Smith, B. A. Soderblom, L. A., Beebe, R., et al. 1981, Science, 212, 163

Smith, B. A., Soderblom, L. A., Batson, R., et al. 1982, Science, 215, 504

Smith, P. H., Lemmon, M. T., Lorenz, R. D., et al. 1996, Icarus, 119, 336

Sromovsky, L. A., Suomi, V. E., Pollack, J. B., et al. 1981, Nature, 292, 698

Sromovsky, L. A., \& Fry, P. M. 1989, Bull. Am. Astron. Soc., 21, 959

Sromovsky, L. A., Irwin, P. G. J., \& Fry, P. M. 2006, Near-IR methane absorption in outer planet atmospheres: Improved models of temperature dependence and implications for Uranus cloud structure, Icarus, in press

Strong, K. Taylor, F. W., Calcutt, S. B., et al. 1993, J. Quant. Spectrosc. Radiat. Transfer, 50, 363

Tobie, G., Grasset, O., Lunine, J. I., et al. 2005, Icarus, 175, 496

Tokano, T., Neubauer, F. M., Laube, M., \& McKay, C. P. 2001, Icarus, 153, 130

Tomasko, M. G., \& Smith, P. H. 1982, Icarus, 51, 65

Tomasko, M. G., Archinal, B., Becker, T., et al. 2005, Nature, 7069, 765

Toon, O. B., McKay, C. P., Griffith, C. A., \& Turco, R. P. 1992, Icarus, 95, 24

Tran, T.-T., \& Rannou, P. 2004, Comparing 3D spherical Monte-Carlo and 2stream parallel plane simulation of far-field backscattering image of Titan. Notes du Pôle de Planétologie de l'Institut Pierre-Simon Laplace, 2

Waite, J. H., Grodent, D. Jr., Mauk, B. M., et al. 2000, Adv. Space Res., 26, 1453

West, R. A.. Hart, H., Simmons, K. E., et al. 1983, J. Geophys. Res., 88, 8699

West, R. A., \& Smith, P. H. 1991, Icarus, 90, 330

Young, E. F., Rannou, P., McKay, C. P., et al. 2002, The Astron. J., 123, 3473 